\title{
Validation of default probability models: A stress testing approach is
}

\author{
Fábio Yasuhiro Tsukahara ${ }^{\mathrm{a}}$, Herbert Kimura ${ }^{\mathrm{b}}$, Vinicius Amorim Sobreiro ${ }^{\mathrm{b}, *}$, Juan Carlos Arismendi Zambrano ${ }^{\mathrm{c}, \mathrm{d}}$ \\ a Midway Finance, 500 Leão XIII, São Paulo, São Paulo, 02526-000, Brazil \\ b University of Brasília, Department of Management, Campus Darcy Ribeiro, Brasília, Federal District, 70910-900, Brazil \\ c Department of Economics, Federal University of Bahia, Rua Barão de Jeremoabo, 668-1154, Salvador, Brazil \\ d ICMA Centre, Henley Business School, University of Reading, Whiteknights, Reading RG6 6BA, United Kingdom
}

\section{A R T I C L E I N F O}

\section{Article history:}

Received 15 July 2015

Received in revised form 31 May 2016

Accepted 28 June 2016

Available online 8 July 2016

\section{Keywords:}

Portfolio

Credit risk

Banking

Default probability

Validation techniques

\begin{abstract}
A B S T R A C T
This study aims to evaluate the techniques used for the validation of default probability (DP) models. By generating simulated stress data, we build ideal conditions to assess the adequacy of the metrics in different stress scenarios. In addition, we empirically analyze the evaluation metrics using the information on 30,686 delisted US public companies as a proxy of default. Using simulated data, we find that entropy based metrics such as measure $M$ are more sensitive to changes in the characteristics of distributions of credit scores. The empirical sub-samples stress test data show that $A U R O C$ is the metric most sensitive to changes in market conditions, being followed by measure $M$. Our results can help risk managers to make rapid decisions regarding the validation of risk models in different scenarios.
\end{abstract}

(c) 2016 Elsevier Inc. All rights reserved.

\section{Introduction}

In summary, the Basel II Accord allows banks to develop internal models for measuring risk (BCBS, 2006; Kiefer, 2009) and the Basel III Accord aims to enhance the stability of the financial system by strengthening risk coverage and highlighting the importance of onand off-balance sheet risks, including derivatives exposure (BCBS, 2011). In addition, the Accords also require validation of risk models to determine, ${ }^{1}$ qualitatively and quantitatively, the models' performance and adherence to the institution's goals. In this context, Stein (2007) states that the validation process is of great importance, since it allows the benefits generated by the use of risk models to be fully obtained. However, effectively validating risk models is still a great challenge, because this is a recent aspect of banking regulation and the primary methods are still under development. In particular, credit model validation has major impediments, i.e., the small

\footnotetext{
is This document is a collaborative effort.

* Corresponding author.

E-mail addresses: fabioyat@gmail.com (F.Y. Tsukahara), herbert.kimura@gmail.com

(H. Kimura), sobreiro@unb.br (V.A. Sobreiro), j.arismendi@icmacentre.ac.uk

(J.C.A. Zambrano).

${ }^{1}$ For financial institutions to be able to use their internal models to calculate capital requirements, the Basel II Accord requires that the models be validated by an independent internal team. For this internal validation process it is necessary to develop techniques to consistently assess the performance of the models used. In this study, we present some validation techniques that are widely used in the financial market for assessment of models, such as $K S$ and $A R$, and other less traditional measures, such as CIER and measure $M$.
}

number of observations to accurately evaluate model performance (Lopez \& Saidenberg, 2000). ${ }^{2}$

Many validation techniques of models for bank risk management have been proposed or submitted in recent years, for market risk (Alexander \& Sheedy, 2008; Boucher, Daníelsson, Kouontchou, \& Maillet, 2014), credit risk (Lopez \& Saidenberg, 2000; Agarwal \& Taffler, 2008), and model risk (Kerkhof \& Melenberg, 2004; Alexander \& Leontsinis, 2011; Alexander \& Sarabia, 2012; Colletaz, Hurlin, \& Pérignon, 2013). Blöchlinger (2012) presents a methodology where the validation of default probability $(D P)$ is produced over credit rating methodologies. Medema, Koning, and Lensink (2009) proposes a practical methodology for validation of statistical models of $D P$ for portfolio of individual loans where no credit rating can be associated. However, there are no studies that attempt to identify or guide managers regarding which model is most appropriate for a given situation. With regard to the methods for estimating credit risk parameters, DP models are, according to BCBS (2005a), those that have the most developed validation

\footnotetext{
2 The growth of credit activity is an important aspect of economic development, because credit is a major source of funds for private and public organizations (Hagedoorn, 1996). However, increases in credit supply bring more exposure to credit risk and, in extreme cases, overreliance on credit can compromise the stability of the financial system (Abou-El-Sood, 2015; Arnold, Borio, Ellis, \& Moshirian, 2012). Economic crises, such as the one in 2008, indicate a need for greater control and regulation of financial institutions by supervisors and for the development of risk management models. In this context, the Basel I, II, and III Accords are examples of how regulatory agencies are concerned with securing a solid international financial system; they are dynamically adjusting their requirements due to an ever-changing economic environment.
} 
methodology. Tasche (2006) separates the performance validation process for these models into two parts, discriminative ability and calibration.

Our contribution to the literature is twofold. First we evaluate the stress test the adequacy of the primary models for risk management and thereby support the decision-making of managers regarding the model selection process. More specifically, we present the characteristics and main properties of different techniques that allow a manager to choose among classic validation models, such as the KolmogorovSmirnov (KS) statistic, Accuracy Ratio (AR), and Brier Score, and newer validation models, such as the Conditional Information Entropy Ratio (CIER) and Measure $M$. The stress test simulation ${ }^{3}$ is carried out in two phases: (i) an assessment of the performance of models to separate good and bad borrowers among the risk groups is performed, (ii) the accuracy of the probabilities estimated by each model is evaluated. ${ }^{4}$ The models were applied to credit portfolios, which were compiled using Monte Carlo simulations, to identify good and bad borrowers and how the characteristics (e.g., dependencies or moments) of these portfolios impacted the results of the models. According to Zott (2003), when there are significant limitations on gathering empirical data and variables have complex interrelationships, simulation may be useful and can actually lead to superior insights into the phenomenon. ${ }^{5}$ The objective of this study is not to exhaustively explore the subject but rather to enable managers to quickly identify a small number of optimal models.

Second, we analyze the default probability validation metrics using controlled sub-samples of market data. Our empirical stress analysis includes financial data of from 30,686 public US firms from 1950 and 2014 , using delisting information as a proxy for default. We develop a methodology that aggregates different groups of years by high-low mean, variance, and correlation related to the financial explanatory variables. Although using empirical data does not allow as total control as using simulated data, the method gives some control over the distribution of credit scores and dependence among variables. Therefore, we can also analyze the behavior of $D P$ evaluation metrics on empirical sub-sample data.

In the case of controlled stress simulations, for independent explanatory variables, we found that (i) the measure $M$ was the only metric able to detect changes in the mean of the explanatory variables, ${ }^{6}$ while there was no metric sensitive to changes in the variances; (ii) all metrics were very sensitive to the number of observations; therefore, the study can help in the validation of models for the retail and large corporations segment. In the case of controlled stress simulations, for dependent explanatory variables, we found that (i) the only metric that captured a performance decrease for both increases and decreases in the correlation parameter was measure $M$, all other measures exhibited an increase in performance as the strength of the correlation was decreased; (ii) modeling using the $T$ copula and Gaussian copula provided no difference in the sensitivity results of the metrics.

The remainder of this study is structured as follows: in Section 2, a literature review of credit is presented; Section 3 and 4 address the aspects used to compare the models and their results; Section 5 presents a empirical application; in Section 6, the primary conclusions are presented and discussed.

\footnotetext{
${ }^{3}$ Simulated portfolios to study credit risk have been explored in the literature. For instance, Kalkbrener, Lotter, and Overbeck (2004) develops an importance sampling Monte Carlo technique to study capital allocation for credit portfolios and Jobst and Zenios (2005) use simulation to analyze the sensitiveness of credit portfolio values to default probability, recovery rates, and migration of ratings. In addition, Hlawatsch and Ostrowski (2011) study loss given default based on simulated datasets to analyze the synthesized loan portfolios.

4 Since there are many classification techniques used for credit scoring (Baesens et al., 2003), performance measurement is necessary to assess model adequacy (Verbraken et al., 2014).

${ }^{5}$ Davis, Eisenhardt, and Bingham (2007) presents a reference to the theory developed using simulation methods.

${ }^{6}$ Explanatory variables are any variables that can lead to a causal explanation of the relationships in default, such as the ones included in the Z-score of Altman (1968).
}

\section{Literature review}

The Basel II Accord aims to improve the awareness of the financial institutions regarding their credit risk (Hakenes \& Schnabel, 2011). The Basell II Accord first pillar aims to guide the calculation of minimum capital requirements, i.e., it reviews the main ideas presented in the Basel I Accord. The minimum capital requirement is calculated based on the Internal Rating Based (IRB) method, which is generally estimated internally by a bank based on the following parameters: (i) DP; (ii) Exposure at Default (EAD); (iii) Loss Given Default ( $L G D)$; and (iv) Maturity $(M)$. It is worth noting that in the simplified version of the IRB, it is only necessary to calculate the $D P$ value because the other parameters are defined by regulatory bodies. From this point of view, the calculation of $D P$ becomes crucial.

\subsection{Validation tests for default probability models}

Two of the most used validation tests are the Cumulative Accuracy Profile (CAP) curve and AR developed by Sobehart, Keenan, and Stein (2000a). Their calculation is performed by ranking all parties based on the scores estimated by the model. Once ranked, for a certain cutoff score, it is possible to identify the fraction of defaults and non-defaults with scores that are less than the cutoff score. The CAP curve is obtained by calculating these fractions for all possible cutoff points, as shown in Fig. 1.

According to Engelmann, Hayden, and Tasche (2003), the AR can be defined by:

$A R=\frac{a_{R}}{a_{P}}$,

where $a_{R}, a_{P}$ are the areas defined in Fig. 1. The closer the $A R$ is to one, the greater the discriminative ability of the model.

The Receiver Operating Characteristic (ROC) curve and the area under the $R O C$ curve are other widely used validation measures developed by Tasche (2006). The ROC curve is obtained by plotting $H R(C)$ versus $F A R(C)$, where $H R(C)$ is the hit rate and $F A R(C)$ the false alarm rate at score $C$. According to Engelmann et al. (2003), the higher the area under the ROC curve of the model, the better the performance. Considering the ideal situation, i.e., an $R O C$ area equal to 1 , the area may be calculated using Eq. (2):

$A U R O C=\int_{0}^{1} H R(F A R) d(F A R)$.

The Pietra Index developed by Pietra $(1915)^{7}$ is a widely used index, whose geometric interpretation corresponds to half of the shortest distance between the ROC curve and the diagonal. This index can be calculated as:

$P I=\frac{\sqrt{2}}{4} \max _{c}|H R(C)-F A R(C)|$

Sobehart et al. (2000a) defined the CIER measure according to:

CIER $=\frac{H_{0}(P)-H_{1}}{H_{0}(P)}$

where $H_{0}(P), H_{1}$ are entropy functions developed by Jaynes (1957) and related to Kullback-Leibler $(K L)$ distance, with the purpose of finding a function with conditions of continuity, monotonicity, and composition law, that represents the uncertainty of a probability distribution. Keenan and Sobehart (1999) defined the measure $H_{0}(p)$ as the entropy of a binary event for which $p$ is the default rate of the sample.

\footnotetext{
7 See Eliazar and Sokolov (2010) for a recent economic application.
} 


\section{Cumulative Accuracy Profile}

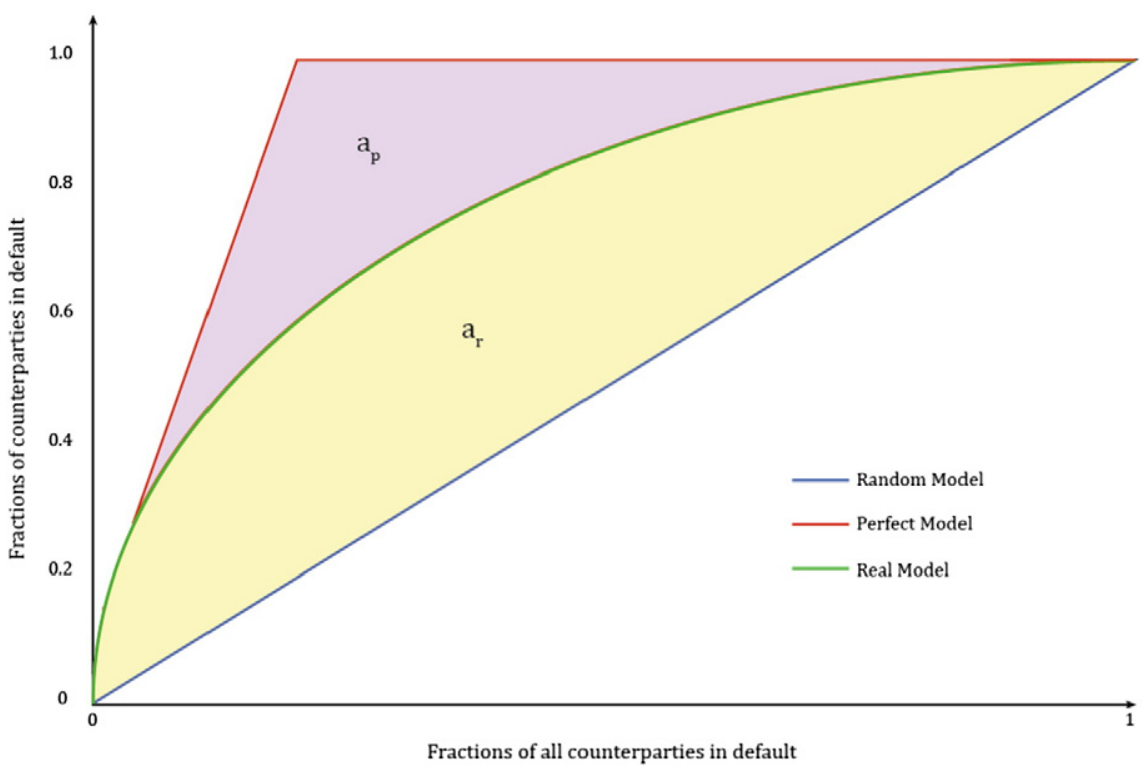

Fig. 1. Cumulative accuracy profile.

The BRIER score was originally proposed by Brier (1950); this metric has the objective of measuring the accuracy of forecasts provided by a given model; and it was initially proposed to measure the accuracy of weather forecasts. The Brier Score can be calculated according to:

BRIER $=\frac{1}{N} \sum_{i=1}^{N}\left(P_{i}-O_{i}\right)^{2}$

where $P_{i}$ corresponds to the probability of occurrence of the event given by the model for the $i$-th component of the sample; and $O_{i}$ corresponds to a binary variable $(1 / 0)$, where one means that the event was observed and zero means that the event was not observed. A perfect $D P$ model would estimate a probability equal to one for observed default events and zero probability for default events that are not observed. Consequently, the Brier Score would be equal to zero, i.e., a Brier Score closer to zero indicates higher accuracy of the model.

Measure $M$ is proposed by Ostrowski and Reichling (2011), and it aims to evaluate the discriminative ability of the default models. Let $\left(a_{D, i}, a_{N D, i}\right)$ be areas of default and non-default, and $\left(r_{D, i}\right.$ and $\left.r_{N D, i}\right)$ hit rates of default and non-default for the $i-$ th rating, (Ostrowski \& Reichling, 2011) defined a measure of the performance of the model:

$m=\sum_{i=1}^{k}\left[H R_{i}\left(r_{D, i}-a_{D, i}\right)+F A R_{i}\left(r_{N D, i}-a_{N D, i}\right)\right]$

where $k$ corresponds to the total number of ratings. Note that this measure is not yet standardized, which precludes direct comparison of two different models. To standardise this measure, the values of $m_{\max }$ and $m_{\min }$ are calculated and the standardized $M$ measure is:

$m_{\min }=\sum_{i=1}^{k} \min \left\{H R_{i}\left(R_{D, i}-a_{D, i}\right), F A R_{i}\left(r_{N D, i}-a_{N D, i}\right)\right\}$,

The value of $\mathfrak{m}$ should be in the range of 0 and 1 , where 1 indicates perfect predictive ability of the model.

According to the studies of Kolmogorov-Smirnov, Lilliefors (1967) presents a procedure to test if a set of $n$ observations is derived from a normal distribution. In a simplified manner, Lilliefors (1967) proposes a hypothesis test for measure $D$ which is the absolute difference between the accumulated distribution function of the sample; and the normal accumulated distribution function with mean and variance equal to those of the sample. To validate credit risk models, it is worth noting that the aim is not to analyze the normality of a distribution but rather to check whether the model can distinguish defaults and non-defaults. For such a purpose, the KS statistic can be used, as described in Joseph (2005), to quantify the greatest distance between the accumulated distribution of defaults and non-defaults. KS can be calculated using:

$K S=\max \left|F_{D}(S)-F_{N D}(S)\right|$,

where $F_{D}$ corresponds to the accumulated distribution function of default cases,

$F_{N D}$ corresponds to the accumulated distribution function of nondefault cases, and $S$ corresponds to the score.

The parameter of information value (IV) proposed by Tasche (2006) measures how default and non-default events are distributed differently among ratings. Let $R_{i}$ be the $i-$ th rating, $p_{D}\left(R_{i}\right)$ the ratio of defaults of the $i$-th rating, and $p_{N D}\left(R_{i}\right)$ the ratio of non-defaults of the $i-t h$ rating. Then, the value of $I V$ can be calculated following (Joseph, 2005) using:

$I V=\sum_{i}\left[p_{D}\left(R_{i}\right)-p_{N D}\left(R_{i}\right)\right] \times \operatorname{In}\left[\frac{p_{D}\left(R_{i}\right)}{p_{N D}\left(R_{i}\right)}\right]$,

It is important to highlight that high IV values indicate high discriminative ability (Tasche, 2006).

\subsection{Studies of the validation of DP models}

Although the process of validation of credit risk models required by Basel II is still relatively new to the global financial market, some studies about model performance measurement techniques had been previously published. Among these studies, the following are noteworthy.

Keenan and Sobehart (1999) presented the following techniques to measure the performance of predictive default models CAP, AR, CIER, and Mutual Information Entropy (MIE). Using a dataset that included data from 9,000 public companies, covering the years of 1989 through 1999, and containing 530 default events, the authors applied a return based model and four additional prediction models (Altman, 1968; Shumway, 2001; Merton, 1974; Sobehart et al., 2000a), the authors were able to conclude that the tests were effective and measured 
distinct aspects of the model. Keenan and Sobehart (1999) emphasized that the CAP curve and the $A R$ measure the discriminative ability of the default model prediction and the CIER and MIE assess whether different models interact by adding information or are simply redundant; Hanley and McNeil (1982) and Engelmann et al. (2003) presented the Receiver Operator Characteristic $(R O C)$ technique and explained its use in the context of validation of rating models. There exists a relationship between between the $A R$ and the area under the ROC curve (AUROC) that can be calculated by:

$A R=2 \times A U R O C-1$,

Karakoulas (2004) presented a validation methodology for credit scoring and DP models for the retail segment and small companies. The author also argued that the KS statistic has the limitation of not referring to where the point of maximum distance occurs and that the $A U R O C$ is more generic regarding this point and therefore better; Joseph (2005) presented a validation methodology based on several tests, and the final evaluation of the model was based on the average performance of the models in these tests. In addition to the $A R, R O C$, $K S$, and Kullback Leibler measures, Joseph (2005) used other measures, such as the mean difference and IV.

Ostrowski and Reichling (2011) found that the AR and AUROC measures can, in certain circumstances, lead to erroneous conclusions and cause low-performance models to be rated well according to these indicators. This observation is in accordance with Engelmann et al. (2003), as the author states that if the distribution of default events is bimodal, a perfect model can have an AUROC equal to that of a random model. Furthermore, Ostrowski and Reichling (2011) proposed another measure called $M$, to measure the performance of the model and applied this new measure in the credit rating models used by the agencies Standard \& Poor's and Moody's. Considering the period from 1982 to 2001, the authors observed that the AUROC measure behaved in a stable manner compared with measure $M$, which exhibited high variability in the measurement of model performance.

\section{Numerical stress test simulation}

Taking into account that different simulated situations lead to distinct behaviors of the metrics it is possible to analyze how the characteristics of the default phenomenon could influence a broad set of evaluation metrics. Consequently, we studied the traditional performance measures like KS, AUROC and AR (BCBS, 2005b; Hand, 2009; Keenan \& Sobehart, 1999; Marshall, Tang, \& Milne, 2010; Ostrowski \& Reichling, 2011; Verbraken, Bravo, Weber, \& Baesens, 2014) as well as other less common metrics like Pietra, BRIER, CIER, Kullback-Leibler (KL), Information Value (IV) and measure $M$ (Joseph, 2005; BCBS, 2005b; Ostrowski \& Reichling, 2011; Izzi, Oricchio, \& Vitale, 2012).

Our analysis of the validation techniques can be divided into two parts:

1. In the first part, good and bad borrower distributions were simulated according to an arbitrary scoring rule and assigned to variable $Y_{B}$. The properties of the distributions were then changed. With this setting, it was possible to analyze the impact of these changes on the values calculated using the validation techniques. This part of the methodology can be summarized by

(a). Generation of the variable $Y_{B}$ of good and bad borrowers using a Monte Carlo simulation approach with a normal distribution to tag subjects into good and bad borrowers;

(b). Generation of explanatory variables $X_{1}, X_{2}$ by a normal distribution with different mean and volatility, depending on whether the subject was tagged as a good or bad borrower in step 1 ; (c). Generation of default variable $Y$ by a gamma distribution;

(d). Association of $X_{i}, i=1,2$ with $Y$ using a bi-stochastic matrix; and,

(e). Calculation of the performance of the entropy-based validation measures by their sensitivity to changes in $X_{1}$ and $X_{2}$.

2 . In the second part of the study logistic models were developed from simulated portfolios that contained a default event and other independent variables. As a consequence, it was possible to analyze how changes in the variables, or in the existing relationships between them, affected the values measured by the techniques studied. This part of the methodology includes

(a). Use of the logistic model to calculate the probability $P$ of the default of a subject depending on $X_{1}$ and $X_{2}$;

(b). Generation of credit scores using $P$, having n-ratings (10 in our numerical simulation) for the classification of the subject;

(c). Calculation of the number of realized defaults from the variable $Y$ obtained in the first part of the methodology; and.

(d). Calculation of the performance of rating-based validation measures by their sensitivity to changes in $X_{1}$ and $X_{2}$.

The methodology presented associated changes in the distribution properties of $X_{1}, X_{2}$, depending on whether is it is a good or bad borrower, with the performance of the techniques for the validation of $D P$. We then analyzed how different changes in distribution parameters and relationships between variables affect the performance of the validation techniques. $^{8}$

\subsection{Normal distribution simulation for good and bad borrowers}

Applying the Monte Carlo simulation technique, different portfolios that contained normal distributions of good and bad borrowers were generated. All portfolios consisted of 30,000 simulations and a bad borrower rate of approximately $10 \%$. The parameters changed in the different portfolios were the mean scores of good and bad borrowers and the deviations of both distributions. Although the deviations were changed, this change was performed on both distributions such that in all portfolios, the deviation of the bad borrower distribution was equal to the deviation of the good borrower distribution. The procedure used to construct the portfolio was as follows:

1. Random classification of the portfolio subjects into good and bad borrowers;

2. Determination of the mean score of the distribution of good borrowers, mean score of the distribution of bad borrowers. and standard deviations of both distributions; and,

3. Assignment of a score to each subject using the Monte Carlo simulation technique

Table 1 presents the parameters used in the different simulated portfolios.

We generated simulated data for the relevant variables to analyze the effects of parameters of the credit score distributions of good and bad borrowers on the validation metrics. Once the simulations were completed, the KS, AUROC, AR, and Pietra Index techniques were applied. Subsequently, each portfolio had its score ranked, and the 30,000 components were distributed into 10 ratings of 3,000 components each. Rating 1 contained the lowest scores, and rating 10 contained the highest scores. Through separation in ratings, it was possible to estimate the values of the CIER, Kullback Leibler and IV validation techniques.

\footnotetext{
8 The use of simulated data makes it possible to have control over the various relationships among variables. Therefore, we can analyze the adequacy of the validation techniques in a controlled environment. When using empirical data from real default situations, the very complexity of the phenomenon can preclude an analysis focused solely on the variables of interest, jeopardizing the study of the validation of models. Nevertheless, we also conducted an empirical analysis aiming to identify the behavior of validation models under real world credit events. Therefore, our study also allows the comparison of results using both simulated and real-world data.
} 
Table 1

Parameters of the normal distributions of score of good and bad borrowers.

\begin{tabular}{lll}
\hline $\begin{array}{l}\text { Mean of score of good } \\
\text { borrowers }\end{array}$ & $\begin{array}{l}\text { Mean of score of bad } \\
\text { borrowers }\end{array}$ & $\begin{array}{l}\text { Standard deviation of score good } \\
\text { and bad borrowers }\end{array}$ \\
\hline 7.5 & 2.0 & $2.0 ; 2.5 ; 3.0$ \\
7.0 & 2.5 & $2.0 ; 2.5 ; 3.0$ \\
6.5 & 3.0 & $2.0 ; 2.5 ; 3.0$ \\
6.0 & 3.5 & $2.0 ; 2.5 ; 3.0$ \\
5.5 & 4.0 & $2.0 ; 2.5 ; 3.0$ \\
\hline
\end{tabular}

3.2. Normal distribution simulation for good borrowers and bimodal distribution simulation for bad borrowers

Similar to portfolios with normal score distributions for good and bad borrowers, portfolios that contained a bimodal distribution for bad borrowers were developed using Monte Carlo simulations, with 30,000 observations per portfolio and a bad borrower rate of $10 \%$. The difference compared with using a normal distribution for bad borrowers is that rather than a normal distribution, the bimodal distribution consists of two normal distributions, one with a mean score that is less than the distribution mean of good borrowers $\left(D M_{1}\right)$ and another with a higher mean score $\left(D M_{2}\right)$. For these portfolios, the distribution deviations of good borrowers, $D M_{1}$ and $D M_{2}$, were kept constant and equal to 1.0, and the distribution mean of good borrowers was kept constant and equal to 5.0. The parameters changed for analysis of the bimodal distributions were the distribution means $D M_{1}$ and $D M_{2}$ and the bimodality intensity, i.e., the number of bad borrowers in $D M_{1}$ and $D M_{2}$. The total number of bad borrowers, i.e., the number of bad borrowers in the two distributions, is approximately equal to $10 \%$ of the portfolio. The procedure used to construct the portfolio contained the following steps:

1. Random classification of the portfolio subjects into good and bad borrowers. When the subject was classified as a bad borrower, a second random classification was performed to determine whether it belonged to distribution $D M_{1}$ or distribution $D M_{2}$;

2. Definition of the mean scores of distributions $D M_{1}$ and $D M_{2}$; and.

3. Assignment of a score to each subject using the Monte Carlo simulation technique

Table 2 presents the parameters used in the different simulated portfolios.

In this analysis we focus on assessing the adequacy of evaluation metrics when the credit score distribution of bad borrowers is bimodal. In particular, we analyze the effects of changing the characteristics of the bimodality of the distribution. The procedure of dividing the portfolio into ratings and the application of validation techniques was performed identically to that for the portfolios with normal distributions for good or bad borrowers.

Table 2

Parameters of the bimodal distribution of score of bad borrowers.

\begin{tabular}{lll}
\hline Mean $D M_{1}$ score & Mean $D M_{2}$ score & Percentage $D M_{1} / D M_{2}$ \\
\hline 7.5 & 2.5 & $90 \% \mid 10 \%$ \\
7.0 & 3.0 & $80 \% \mid 20 \%$ \\
6.5 & 3.5 & $70 \% \mid 30 \%$ \\
6.0 & 4.0 & $60 \% \mid 40 \%$ \\
5.5 & 4.5 & $50 \% \mid 50 \%$ \\
\hline
\end{tabular}

Note: The first and second columns depict the mean the third column shows the percentage of bad borrows in the neighborhood of each of mode of the bimodal distribution.

\subsection{Generation of simulated portfolios}

For the construction of the portfolios used to obtain the logistic models, the values of the dependent and explanatory variables were simulated. In the case of default probability models, some examples of explanatory variables are the ratio of sales to total assets and the ratio of retained earnings to total assets, which are used in the Altman Z-score model Altman (1968). Other examples are the rate of indebtedness and economic sector for the corporate credit segment and the income, age, and occupation of an individual for the credit segments that are related to individuals.

For the construction of the dependent variable (default), random values were simulated from a gamma distribution with parameters $k=1$ and $\theta=0.1$. Then, for each gamma distribution value simulated, a random value was generated from a uniform distribution [0-1]. If the value of the uniform distribution was less than the value of the gamma distribution, the dependent variable Y would have value 1 (Default); otherwise, it would have value 0 (non-default). The explanatory variables $X_{1}$ and $X_{2}$ were built using randomly simulated values of normal distributions. Variable $X_{1}$ has mean equal to 7.0 and deviation equal to 2.0. Variable $X_{2}$ has mean equal to 20.0 and deviation equal to 4.0 .

\subsubsection{Dependence among variables}

For the generation of explanatory variables with dependence, the copula method was used. This method allows, based on Sklar's theorem, us to formulate joint distributions with several types of dependence. Nelsen (1999) states that copulas are functions that join or couple joint distribution functions to their unidimensional marginal distribution functions. Thus, in this study, portfolios in which $X_{1}$ and $X_{2}$ were independent and dependent according to Gaussian copulas were used.

\subsubsection{Association between the dependent variable and independent variables}

Once the explanatory variables with dependencies are simulated, it is necessary to simulate the default events and use a method that allows us to associate the default events with explanatory variables. This association was made based on a method that uses bi-stochastic matrices. Bi-stochastic matrices can be seen as a discrete version of a copula. Briefly, this method, which is based on that of Hlawatsch and Ostrowski (2011), consists of separating both the dependent and explanatory simulated variables into groups and associating the groups according to a dependency structure. Hence, according to the proposition by Hlawatsch and Ostrowski (2011), the steps used to associate a dependent variable and an independent variable with a negative causal relationship, considering $Y$ as the dependent variable and $X$ as the explanatory variable, are as follows:

1. Sort each variable and separate them in blocks such that the first block contains the lowest values and the last block has the highest values;

2. Next, a bi-stochastic matrix $M$ is constructed with elements $m_{(i, j)}$ that represent the probability of observing an element of the $i-t h$ block of $Y$ associated with an element of the $j-$ th block of $X$. The indices $i$ and $j$ are natural numbers and range from one to the number of groups (in this study, five groups were used), and the parameters $m_{(i, j)}$, that comply with the conditions given by

$\sum_{i=1}^{5} m_{i, j}=1, \quad \sum_{j=1}^{5} m_{i, j}=1$.

Although it may seen counter-intuitive to use a discrete dependence measure to associate two continuous variables, it is possible, considering that there will be some error produced by discretization of the dependence of the continuous variables. This error is reduced by increasing the size of the bi-stochastic matrix. 
Table 3

Validation techniques applied to the normal distributions of good and bad borrowers.

\begin{tabular}{|c|c|c|c|c|c|c|c|c|c|}
\hline $\begin{array}{l}\text { Standard Deviation of scores Good and Bad } \\
\text { Borrowers }\end{array}$ & $\begin{array}{l}\text { Mean of scores of Good } \\
\text { Borrowers }\end{array}$ & $\begin{array}{l}\text { Mean of scores of Bad } \\
\text { Borrowers }\end{array}$ & KS & AUROC & $\mathrm{AR}$ & Pietra & CIER & $\mathrm{KL}$ & IV \\
\hline \multirow[t]{5}{*}{2.0} & 7.5 & 2.5 & 0.766 & 0.952 & 0.904 & 0.271 & 0.500 & 0.161 & 4.690 \\
\hline & 7.0 & 3.0 & 0.660 & 0.911 & 0.822 & 0.233 & 0.370 & 0.119 & 3.281 \\
\hline & 6.5 & 3.5 & 0.524 & 0.842 & 0.683 & 0.185 & 0.231 & 0.075 & 1.834 \\
\hline & 6.0 & 4.0 & 0.369 & 0.746 & 0.492 & 0.130 & 0.108 & 0.034 & 0.821 \\
\hline & 5.5 & 4.5 & 0.205 & 0.641 & 0.282 & 0.072 & 0.035 & 0.011 & 0.254 \\
\hline \multirow[t]{5}{*}{2.5} & 7.5 & 2.5 & 0.616 & 0.888 & 0.777 & 0.218 & 0.315 & 0.101 & 2.626 \\
\hline & 7.0 & 3.0 & 0.533 & 0.843 & 0.686 & 0.188 & 0.233 & 0.076 & 1.899 \\
\hline & 6.5 & 3.5 & 0.399 & 0.764 & 0.528 & 0.141 & 0.129 & 0.041 & 0.972 \\
\hline & 6.0 & 4.0 & 0.292 & 0.695 & 0.389 & 0.103 & 0.066 & 0.022 & 0.493 \\
\hline & 5.5 & 4.5 & 0.153 & 0.601 & 0.202 & 0.054 & 0.018 & 0.006 & 0.130 \\
\hline \multirow[t]{5}{*}{3.0} & 7.5 & 2.5 & 0.508 & 0.823 & 0.646 & 0.180 & 0.204 & 0.067 & 1.558 \\
\hline & 7.0 & 3.0 & 0.420 & 0.774 & 0.548 & 0.148 & 0.139 & 0.046 & 1.043 \\
\hline & 6.5 & 3.5 & 0.308 & 0.705 & 0.410 & 0.109 & 0.075 & 0.025 & 0.541 \\
\hline & 6.0 & 4.0 & 0.209 & 0.644 & 0.389 & 0.074 & 0.035 & 0.012 & 0.262 \\
\hline & 5.5 & 4.5 & 0.116 & 0.577 & 0.154 & 0.041 & 0.010 & 0.003 & 0.071 \\
\hline
\end{tabular}

Table 4

Validation techniques applied to the normal distribution of good borrowers and bimodal distribution.

\begin{tabular}{|c|c|c|c|c|c|c|c|c|c|c|}
\hline Ratio of bad borrowers $\left(D M_{1}\right)$ & Ratio of bad borrowers $\left(D M_{2}\right)$ & Mean $D M_{1}$ & Mean $D M_{2}$ & KS & AUROC & AR & Pietra & CIER & $\mathrm{KL}$ & IV \\
\hline \multirow[t]{5}{*}{$90 \%$} & \multirow[t]{5}{*}{$10 \%$} & 7.5 & 2.5 & 0.708 & 0.875 & 0.750 & 0.250 & 0.467 & 0.152 & 3.901 \\
\hline & & 7.0 & 3.0 & 0.594 & 0.838 & 0.676 & 0.210 & 0.315 & 0.104 & 2.395 \\
\hline & & 6.5 & 3.5 & 0.460 & 0.773 & 0.546 & 0.163 & 0.173 & 0.057 & 1.229 \\
\hline & & 6.0 & 4.0 & 0.323 & 0.710 & 0.419 & 0.114 & 0.086 & 0.028 & 0.601 \\
\hline & & 5.5 & 4.5 & 0.165 & 0.611 & 0.222 & 0.058 & 0.021 & 0.007 & 0.151 \\
\hline \multirow[t]{5}{*}{$80 \%$} & \multirow[t]{5}{*}{$20 \%$} & 7.5 & 2.5 & 0.616 & 0.780 & 0.561 & 0.218 & 0.413 & 0.136 & 3.363 \\
\hline & & 7.0 & 3.0 & 0.523 & 0.754 & 0.507 & 0.185 & 0.277 & 0.089 & 2.092 \\
\hline & & 6.5 & 3.5 & 0.415 & 0.720 & 0.440 & 0.147 & 0.161 & 0.053 & 1.141 \\
\hline & & 6.0 & 4.0 & 0.273 & 0.666 & 0.331 & 0.097 & 0.063 & 0.020 & 0.438 \\
\hline & & 5.5 & 4.5 & 0.123 & 0.580 & 0.160 & 0.043 & 0.012 & 0.004 & 0.085 \\
\hline \multirow[t]{5}{*}{$70 \%$} & \multirow[t]{5}{*}{$10 \%$} & 7.5 & 2.5 & 0.524 & 0.684 & 0.369 & 0.185 & 0.366 & 0.118 & 3.011 \\
\hline & & 7.0 & 3.0 & 0.435 & 0.668 & 0.335 & 0.154 & 0.247 & 0.079 & 1.889 \\
\hline & & 6.5 & 3.5 & 0.322 & 0.638 & 0.276 & 0.114 & 0.128 & 0.042 & 0.902 \\
\hline & & 6.0 & 4.0 & 0.225 & 0.612 & 0.224 & 0.079 & 0.049 & 0.016 & 0.343 \\
\hline & & 5.5 & 4.5 & 0.102 & 0.561 & 0.123 & 0.036 & 0.008 & 0.003 & 0.057 \\
\hline \multirow[t]{5}{*}{$60 \%$} & \multirow[t]{5}{*}{$40 \%$} & 7.5 & 2.5 & 0.446 & 0.607 & 0.215 & 0.158 & 0.344 & 0.112 & 2.770 \\
\hline & & 7.0 & 3.0 & 0.365 & 0.586 & 0.172 & 0.129 & 0.246 & 0.081 & 1.826 \\
\hline & & 6.5 & 3.5 & 0.250 & 0.560 & 0.120 & 0.088 & 0.115 & 0.038 & 0.812 \\
\hline & & 6.0 & 4.0 & 0.154 & 0.550 & 0.100 & 0.054 & 0.035 & 0.011 & 0.245 \\
\hline & & 5.5 & 4.5 & 0.063 & 0.523 & 0.047 & 0.022 & 0.005 & 0.002 & 0.034 \\
\hline \multirow[t]{5}{*}{$50 \%$} & \multirow[t]{5}{*}{$50 \%$} & 7.5 & 2.5 & 0.369 & 0.507 & 0.013 & 0.130 & 0.368 & 0.120 & 3.123 \\
\hline & & 7.0 & 3.0 & 0.294 & 0.509 & 0.018 & 0.104 & 0.233 & 0.075 & 1.762 \\
\hline & & 6.5 & 3.5 & 0.205 & 0.505 & 0.009 & 0.073 & 0.112 & 0.036 & 0.793 \\
\hline & & 6.0 & 4.0 & 0.111 & 0.493 & -0.013 & 0.039 & 0.033 & 0.011 & 0.230 \\
\hline & & 5.5 & 4.5 & 0.041 & 0.497 & -0.005 & 0.015 & 0.004 & 0.001 & 0.029 \\
\hline
\end{tabular}

As an example of the application of this technique, suppose that a matrix $M$ is given by:

$M=\left(\begin{array}{lllll}0.01 & 0.04 & 0.08 & 0.16 & 0.71 \\ 0.04 & 0.07 & 0.18 & 0.55 & 0.16 \\ 0.08 & 0.18 & 0.48 & 0.18 & 0.08 \\ 0.16 & 0.55 & 0.18 & 0.07 & 0.04 \\ 0.71 & 0.16 & 0.08 & 0.04 & 0.01\end{array}\right)$

Once the matrix is built, an observation from the first block of $Y$ and a random number from a uniform distribution [0-1] are selected.
Assuming that the random number is 0.4 and analysing the first line of the matrix, the observation of the explanatory variable $X$ associated with the observation of the dependent variable $Y$, which was previously selected, belongs to the fifth block because 0.4 is greater than $0.01+0.04+0.08+0.16$. If the random number is 0.015 , the $X$ observation would be in the second block. This process is replicated until the elements of the first block of $Y$ are exhausted. Then, the process continues to the second block, and the second matrix line is analyzed. The process follows the same form until all associations are performed, i.e., until all $Y$ values have an associated $X$ value. If the $X$ group selected is empty, that is, all elements have been previously selected, a value from the closest non-empty group is selected. Once the process is

Table 5

Confidence interval for validation metrics obtained from portfolios with independent explanatory variables.

\begin{tabular}{|c|c|c|c|c|c|c|c|c|c|}
\hline Initial sample & KS & AUROC & AR & Pietra Index & Brier & CIER & $\mathrm{KL}$ & IV & $M$ \\
\hline Mean & 0.3856 & 0.7495 & 0.4991 & 0.1363 & 0.0829 & 0.1159 & 0.0373 & 0.9407 & 0.3126 \\
\hline Standard deviation & 0.0113 & 0.0059 & 0.0117 & 0.0040 & 0.0025 & 0.0051 & 0.0019 & 0.0442 & 0.0255 \\
\hline Lower limit (95\% CI) & 0.3620 & 0.7373 & 0.4745 & 0.1280 & 0.0776 & 0.1052 & 0.0334 & 0.8482 & 0.7570 \\
\hline Upper limit (95\% CI) & 0.4092 & 0.7618 & 0.5236 & 0.1447 & 0.0882 & 0.1266 & 0.0413 & 1.0332 & 0.8683 \\
\hline
\end{tabular}


Table 6

Values of validation metrics obtained from change in the mean of $X_{1}$.

\begin{tabular}{llllllllll}
\hline $\begin{array}{l}\text { Mean } \\
\boldsymbol{X}_{1}\end{array}$ & KS & AUROC & AR & $\begin{array}{l}\text { Pietra } \\
\text { Index }\end{array}$ & Brier & CIER & KL & IV & M \\
\hline 5.0 & 0.391 & 0.756 & 0.512 & 0.138 & 0.082 & 0.122 & 0.038 & 0.975 & $0.463^{*}$ \\
5.5 & 0.408 & 0.757 & 0.515 & 0.144 & 0.083 & $0.127^{*}$ & 0.041 & $1.129^{*}$ & $0.522^{*}$ \\
6.0 & 0.381 & 0.749 & 0.499 & 0.135 & 0.085 & 0.118 & 0.039 & 0.926 & $0.638^{*}$ \\
6.5 & 0.382 & 0.742 & 0.483 & 0.135 & 0.083 & 0.107 & 0.034 & 0.377 & $0.734^{*}$ \\
7.5 & 0.392 & 0.747 & 0.495 & 0.139 & 0.083 & 0.113 & 0.036 & 0.928 & 0.306 \\
3.0 & 0.379 & 0.748 & 0.497 & 0.134 & 0.085 & 0.115 & 0.038 & 0.934 & 0.775 \\
3.5 & 0.382 & 0.743 & 0.435 & 0.135 & 0.086 & 0.108 & 0.035 & 0.361 & $0.642^{*}$ \\
9.0 & 0.386 & 0.754 & 0.508 & 0.137 & 0.085 & 0.120 & 0.038 & 0.989 & $0.505^{*}$ \\
\hline
\end{tabular}

completed, a set of observations with a dependency relationship between the explanatory variables and the dependent variable is obtained. If $X_{1}$ and $X_{2}$ are independent, two bi-stochastic matrices are used to perform the association, where one of the matrices associates $Y$ to $X_{1}$ and the other associates $Y$ to $X_{2}$. For cases of dependency between $X_{1}$ and $X_{2}$, three variables, $X_{1}, X_{2}$ and $X_{3}$, are simulated, where $X_{3}$ has a normal distribution with mean zero and deviation 1.0. The correlations between $X_{1}$ and $X_{3}$ and that between $X_{2}$ and $X_{3}$ are set to be 0.7 . The association between $Y, X_{1}$ and $X_{2}$ is performed in two steps. In the first, a bistochastic matrix is used to associate $Y$ to $X_{3}$. For each $X_{3}$ value, there are related $X_{1}$ and $X_{2}$ values because the variables are not independent; thus, in the second step, $Y$ is associated with the $X_{1}$ and $X_{2}$ values that are related to the variable $X_{3}$.

\subsubsection{DP model and separation into ratings}

Once the portfolio was built with the binary variable default event observation $(Y)$ and independent variables $X_{1}$ and $X_{2}$, a DP model was developed using the logistic regression technique. The use of logistic models for the development of score models is very common in the financial markets and in academia (Steenackers \& Goovaerts, 1989; Bensic, Sarlija, \& Zekic-Susac, 2005). Through the logistic regression technique, it is possible to estimate the probability of a default event $(Y=1)$ given a set of $n$ explanatory variables. Defining this probability as being $P\left(Y=1 \mid X_{1}, X_{2}, \ldots, X_{n}\right)=P(X)$, the logistic model can be specified following ( $\mathrm{O}^{\prime}$ Connell, 2006) using and (12):

$\ln \left(\frac{P(X)}{1+P(X)}\right)=\beta_{0}+\beta_{1} X_{1}+\beta_{2} X_{2}+\beta_{n} X_{n}=Z$,

Considering (12), the probability of occurrence of a default event for a set of explanatory variables, $P(X)$, can be obtained using (13):

$P(X)=\frac{\exp \left(\beta_{0}+\beta_{1} X_{1}+\cdots+\beta_{n} X_{n}\right)}{1+\exp \left(\beta_{0}+\beta_{1} X_{1}+\cdots+\beta_{n} X_{n}\right)}=\frac{\exp (Z)}{1+\exp (Z)}$.

Because in the simulated portfolios, two independent variables $\left(X_{1}\right.$ and $X_{2}$ ) were used, $Z$ can be calculated using (14), and the model score is obtained using (15):

$Z=\beta_{0}+\beta_{1} X_{1}+\beta_{2} X_{2}$

Score $=1-\frac{e^{Z}}{1+e^{Z}}=1-P$.

In this case, the higher the probability of default of the subject, the lower the score. The number of defaults estimated for the $k-t h$ rating can be obtained as the sum of the probabilities of default of the elements contained in this rating. Hence, for a rating that contains $m$ elements, the number of estimated defaults is obtained using (16):

$Q D E=\sum_{m}^{i=1} D P_{k i}$

Table 7

Observed and estimated values for each rating for different means of the $X_{1}$ explanatory variable.

\begin{tabular}{|c|c|c|c|c|c|c|c|c|c|c|c|c|c|c|c|c|}
\hline \multirow[t]{2}{*}{ Rating } & \multicolumn{4}{|c|}{ Original (Mean $\boldsymbol{X}_{1}=7.0$ ) } & \multicolumn{4}{|c|}{ Mean $\boldsymbol{X}_{1}=5.0$} & \multicolumn{4}{|c|}{ Mean $\boldsymbol{X}_{1}=7.5$} & \multicolumn{4}{|c|}{ Mean $\boldsymbol{X}_{1}=9.0$} \\
\hline & $E t_{M}$ & $E s t_{B}$ & $O b s_{M}$ & $\mathrm{Obs}_{B}$ & $E s t_{M}$ & $E s t_{B}$ & $\mathrm{Obs}_{B}$ & $\mathrm{Obs}_{B}$ & Est $_{M}$ & $E_{s t_{B}}$ & $O b s_{M}$ & $\mathrm{Obs}_{B}$ & $E s t_{M}$ & $E s t_{B}$ & $O b s_{M}$ & $\mathrm{Obs}_{B}$ \\
\hline 1 & 285 & 715 & 252 & 748 & 175 & 825 & 249 & 751 & 321 & 679 & 246 & 754 & 428 & 572 & 266 & 734 \\
\hline 2 & 182 & 818 & 218 & 782 & 104 & 896 & 244 & 756 & 208 & 792 & 237 & 763 & 297 & 703 & 215 & 785 \\
\hline 3 & 122 & 878 & 138 & 862 & 69 & 931 & 104 & 896 & 142 & 858 & 148 & 852 & 208 & 792 & 110 & 890 \\
\hline 4 & 102 & 898 & 106 & 894 & 57 & 943 & 106 & 894 & 119 & 881 & 111 & 889 & 177 & 823 & 115 & 885 \\
\hline 5 & 77 & 923 & 77 & 923 & 42 & 958 & 77 & 923 & 89 & 911 & 63 & 937 & 134 & 866 & 79 & 921 \\
\hline 6 & 64 & 936 & 76 & 924 & 35 & 965 & 66 & 934 & 74 & 926 & 74 & 926 & 114 & 886 & 70 & 930 \\
\hline 7 & 48 & 952 & 33 & 967 & 26 & 974 & 35 & 965 & 55 & 945 & 41 & 959 & 84 & 916 & 37 & 963 \\
\hline 8 & 39 & 961 & 35 & 965 & 21 & 979 & 31 & 969 & 46 & 954 & 39 & 961 & 71 & 929 & 31 & 969 \\
\hline 9 & 26 & 974 & 16 & 984 & 14 & 986 & 13 & 987 & 30 & 970 & 25 & 975 & 47 & 953 & 13 & 987 \\
\hline 10 & 15 & 985 & 10 & 990 & 8 & 992 & 14 & 986 & 17 & 983 & 7 & 993 & 27 & 973 & 10 & 990 \\
\hline
\end{tabular}

Table 8

Values of validation metrics obtained by changing the variance of the $X_{2}$ explanatory variable.

\begin{tabular}{|c|c|c|c|c|c|c|c|c|c|}
\hline Deviation $X_{2}$ & KS & AUROC & AR & Pietra Index & Brier & CIER & $\mathrm{KL}$ & IV & M \\
\hline 10.00 & $0.346^{*}$ & $0.732^{*}$ & $0.464^{*}$ & $0.122^{*}$ & $0.090^{*}$ & $0.093^{*}$ & $0.032^{*}$ & $0.759^{*}$ & $0.683^{*}$ \\
\hline 8.00 & 0.377 & 0.746 & 0.492 & 0.133 & 0.086 & 0.113 & 0.036 & 1.006 & 0.793 \\
\hline 6.00 & 0.375 & 0.751 & 0.502 & 0.132 & 0.085 & 0.118 & 0.039 & 0.939 & 0.817 \\
\hline 5.00 & 0.399 & 0.756 & 0.513 & 0.141 & 0.087 & 0.120 & 0.040 & 0.963 & 0.810 \\
\hline 4.75 & 0.382 & 0.745 & 0.490 & 0.135 & 0.083 & 0.111 & 0.036 & 0.895 & 0.822 \\
\hline 4.50 & 0.388 & 0.745 & 0.490 & 0.137 & 0.082 & 0.107 & 0.034 & 0.850 & 0.820 \\
\hline 4.25 & 0.385 & 0.743 & 0.487 & 0.136 & 0.087 & 0.110 & 0.037 & 0.867 & 0.796 \\
\hline 3.75 & $0.358^{*}$ & $0.737^{*}$ & $0.474^{*}$ & $0.126^{*}$ & 0.086 & $0.103^{*}$ & 0.034 & $0.806^{*}$ & 0.790 \\
\hline 3.50 & 0.376 & 0.742 & 0.485 & 0.133 & 0.082 & 0.111 & 0.035 & 0.895 & 0.826 \\
\hline 3.23 & 0.377 & 0.744 & 0.489 & 0.133 & 0.084 & 0.108 & 0.035 & 0.854 & 0.860 \\
\hline 3.00 & 0.408 & 0.756 & 0.512 & 0.144 & 0.081 & 0.118 & 0.037 & 0.936 & 0.803 \\
\hline
\end{tabular}


Table 9

Observed and estimated values for each rating when changing the variances of the $X_{2}$ explanatory variable.

\begin{tabular}{|c|c|c|c|c|c|c|c|c|c|c|c|c|c|c|c|c|}
\hline \multirow[t]{2}{*}{ Rating } & \multicolumn{4}{|c|}{ Original (Deviation $\boldsymbol{X}_{2}=4.0$ ) } & \multicolumn{4}{|c|}{ Deviation $\boldsymbol{X}_{2}=10.0$} & \multicolumn{4}{|c|}{ Deviation $\boldsymbol{X}_{2}=6.0$} & \multicolumn{4}{|c|}{ Deviation $\boldsymbol{X}_{2}=3.2$} \\
\hline & $E s t_{M}$ & $E t_{B}$ & $O b s_{M}$ & $O b s_{B}$ & $E t_{M}$ & $E t_{B}$ & $\mathrm{Obs}_{B}$ & $O b s_{B}$ & $E s t_{M}$ & Est $_{B}$ & $O b s_{M}$ & $\mathrm{Obs}_{B}$ & $E s t_{M}$ & $E s t_{B}$ & $O b s_{M}$ & $O b s_{B}$ \\
\hline 1 & 285 & 715 & 252 & 748 & 478 & 522 & 264 & 736 & 346 & 654 & 285 & 715 & 262 & 738 & 264 & 736 \\
\hline 2 & 182 & 818 & 218 & 782 & 275 & 725 & 221 & 779 & 206 & 794 & 237 & 763 & 172 & 828 & 225 & 775 \\
\hline 3 & 122 & 878 & 138 & 862 & 162 & 838 & 108 & 892 & 134 & 866 & 105 & 895 & 119 & 881 & 126 & 874 \\
\hline 4 & 102 & 898 & 106 & 894 & 122 & 878 & 126 & 874 & 109 & 891 & 116 & 884 & 100 & 900 & 116 & 884 \\
\hline 5 & 77 & 923 & 77 & 923 & 79 & 921 & 80 & 920 & 77 & 923 & 88 & 912 & 76 & 924 & 87 & 913 \\
\hline 6 & 64 & 936 & 76 & 924 & 61 & 939 & 80 & 920 & 63 & 937 & 72 & 928 & 65 & 935 & 67 & 933 \\
\hline 7 & 48 & 952 & 33 & 967 & 39 & 961 & 54 & 946 & 44 & 956 & 48 & 952 & 49 & 951 & 47 & 953 \\
\hline 8 & 39 & 961 & 35 & 965 & 29 & 971 & 38 & 962 & 35 & 965 & 39 & 961 & 40 & 960 & 39 & 961 \\
\hline 9 & 26 & 974 & 16 & 984 & 16 & 984 & 24 & 976 & 23 & 977 & 19 & 981 & 27 & 973 & 19 & 981 \\
\hline 10 & 15 & 985 & 10 & 990 & 7 & 993 & 17 & 983 & 12 & 988 & 10 & 990 & 16 & 984 & 13 & 987 \\
\hline
\end{tabular}

Table 10

Values of metrics obtained by changing the bi-stochastic matrices.

\begin{tabular}{|c|c|c|c|c|c|c|c|c|c|}
\hline Matrices & KS & AUROC & AR & Pietra Index & Brier & CIER & $\mathrm{KL}$ & IV & M \\
\hline$M 2 \& M_{2}^{\prime}$ & 0.3706 & 0.7415 & 0.4830 & 0.1310 & 0.0845 & 0.1065 & 0.0346 & $0.843^{*}$ & 0.8132 \\
\hline M3 \& $M_{3}^{\prime}$ & $0.332^{*}$ & $0.719^{*}$ & $0.438^{*}$ & $0.117^{*}$ & 0.0853 & $0.085^{*}$ & $0.027^{*}$ & $0.664^{*}$ & 0.8497 \\
\hline$M 4 \& M_{4}^{\prime}$ & $0.300^{*}$ & $0.694^{*}$ & $0.389^{*}$ & $0.106^{*}$ & 0.0863 & $0.066^{*}$ & $0.021^{*}$ & $0.522^{*}$ & 0.8371 \\
\hline$M 5 \& M_{5}^{\prime}$ & $0.190^{*}$ & $0.631^{*}$ & $0.262^{*}$ & $0.067^{*}$ & $0.088^{*}$ & $0.030^{*}$ & $0.009^{*}$ & $0.222^{*}$ & $0.748^{*}$ \\
\hline
\end{tabular}

\subsection{Studies performed}

\subsubsection{Independent explanatory variables}

The processes used for generation and analysis of portfolios whose explanatory variables are independent were the following:

1. Generation of 20 simulated portfolios using the bi-stochastic matrices $M_{1}$ and $M_{1}$, to associate $X_{1}$ and $X_{2}$, respectively, to $Y$;

2. Development of logistic models from simulated portfolios;

3. Application of validation techniques on the 20 models developed and definition of a confidence interval for each technique;

4. Choice of one of the 20 models to be used in the samples with changed parameters;

5. Application of the chosen model and validation techniques to simulated portfolios, where the following parameters were varied

- The mean of variable $X_{1}$;

- The variance of variable $X_{2}$; and.

- The stochastic matrixes $B_{i}$ that relate $X_{1}$ and $X_{2}$ to the dependent variable $Y$.

The objective of the analysis is to identify how changes in the characteristics of independent variables affect the model performance and how each technique responds to these changes. Another important aspect is the sensitivity of the validation techniques to changes in the dependencies between dependent and explanatory variables, i.e., how each technique responds to changes in the bi-stochastic matrices.
3.4.2. Explanatory variables with dependencies simulated via Gaussian copulas

For the case in which the dependencies among explanatory variables were simulated using Gaussian copulas, the following process was performed:

1. Model with zero correlation between $X_{1}$ and $X_{2}$ :

- Generation of 20 simulated portfolios with zero correlation between $X_{1}$ and $X_{2}$ using the bi-stochastic matrix $M_{1}$ for association between explanatory variables and $Y$;

- Development of logistic models from simulated portfolios;

- Application of the validation techniques to the 20 models developed and determination of a confidence interval for each technique;

- Choice of one of the 20 models to be used in samples with changed parameters; and.

- Application of the chosen model and validation techniques to the simulated portfolios, where the correlation parameter between $X_{1}$ and $X_{2}$ was varied.

2. Model with 0.5 correlation between $X_{1}$ and $X_{2}$ :

- Generation of 20 simulated portfolios with a correlation of 0.5 between $X_{1}$ and $X_{2}$ using the bi-stochastic matrix $M_{1}$ for the association between explanatory variables and $Y$;

- Development of logistic models from simulated portfolios;

- Application of the validation techniques to the 20 models developed and determination of a confidence interval for each technique;

- Choice of one of the 20 models to be used in the samples with changed parameters; and.

Table 11

Observed and estimated values for each rating when changing the bi-stochastic matrices.

\begin{tabular}{|c|c|c|c|c|c|c|c|c|c|c|c|c|}
\hline \multirow[t]{2}{*}{ Rating } & \multicolumn{4}{|c|}{ Original $\left(M_{1} \& M_{1}^{\prime}\right)$} & \multicolumn{4}{|c|}{$M_{3} \& M_{3}^{\prime}$} & \multicolumn{4}{|c|}{$M_{5} \& M_{5}^{\prime}$} \\
\hline & $E s t_{M}$ & $E s t_{B}$ & $O b s_{M}$ & $O b s_{B}$ & $E s t_{M}$ & $E t_{B}$ & $O b s_{M}$ & $O b s_{B}$ & $E s t_{M}$ & $E t_{B}$ & $O b s_{M}$ & $O b s_{B}$ \\
\hline 1 & 285 & 715 & 252 & 748 & 270 & 730 & 249 & 751 & 234 & 766 & 199 & 801 \\
\hline 2 & 182 & 818 & 218 & 782 & 170 & 830 & 198 & 802 & 146 & 854 & 140 & 860 \\
\hline 3 & 122 & 878 & 138 & 862 & 120 & 880 & 120 & 880 & 114 & 886 & 107 & 893 \\
\hline 4 & 102 & 898 & 106 & 894 & 100 & 900 & 131 & 869 & 93 & 907 & 120 & 880 \\
\hline 5 & 77 & 923 & 77 & 923 & 77 & 923 & 85 & 915 & 76 & 924 & 98 & 902 \\
\hline 6 & 64 & 936 & 76 & 924 & 64 & 936 & 83 & 917 & 64 & 936 & 88 & 912 \\
\hline 7 & 48 & 952 & 33 & 967 & 48 & 952 & 43 & 957 & 53 & 947 & 74 & 926 \\
\hline 8 & 39 & 961 & 35 & 965 & 40 & 960 & 40 & 960 & 43 & 957 & 75 & 925 \\
\hline 9 & 26 & 974 & 16 & 984 & 28 & 972 & 33 & 967 & 33 & 967 & 59 & 941 \\
\hline 10 & 15 & 985 & 10 & 990 & 16 & 984 & 18 & 982 & 19 & 981 & 40 & 960 \\
\hline
\end{tabular}


Table 12

Confidence interval obtained from simulated portfolios with zero correlation between $X_{1}$ and $X_{2}$.

\begin{tabular}{|c|c|c|c|c|c|c|c|c|c|}
\hline Initial sample & $\mathrm{RS}$ & AUROC & AR & Pietra index & Brier & CIER & $\mathrm{KL}$ & IV & M \\
\hline Mean & 0.3815 & 0.7434 & 0.4868 & 0.1349 & 0.0850 & 0.1076 & 0.0352 & 0.8402 & 0.805114 \\
\hline Standard deviation & 0.0148 & 0.0093 & 0.0186 & 0.0052 & 0.0022 & 0.0090 & 0.0034 & 0.0831 & 0.031882 \\
\hline Lower limit (95\% CI) & 0.3506 & 0.7239 & 0.4478 & 0.1240 & 0.0804 & 0.0887 & 0.0280 & 0.6662 & 0.738385 \\
\hline Upper limit (95\% CI) & 0.4124 & 0.7629 & 0.5258 & 0.1458 & 0.0895 & 0.1266 & 0.0424 & 1.0141 & 0.871843 \\
\hline
\end{tabular}

- Application of the chosen model and validation techniques to the simulated portfolios, where the correlation parameter between $X_{1}$ and $X_{2}$ was varied.

According to these variables, it is possible to identify how the performance of a model that was developed when there was no correlation among explanatory variables is affected by the emergence of this dependency. It is also possible to evaluate how the performance of a developed model, considering the dependencies, is affected if the dependencies are changed over time.

\section{Results of simulations}

To obtain the results of application of the techniques on the logistic models, modelling routines and procedures for generation of variables with or without dependencies were developed in the $R$ language and used in addition to the validation techniques that were implemented in Visual Basic Application (VBA) software. The results for the score distribution simulation were obtained by implementing the simulations and validation techniques using VBA.

\subsection{Simulation of score distributions}

A total of 15 bases that contained simulations of cases in which good and bad borrowers had normal distributions were generated. The parameters that varied among the portfolios were the means and deviations of the distributions. For cases in which the distribution was normal for good borrowers and bimodal for bad borrowers, 25 simulated bases were generated, and the parameters that were varied among the portfolios were the means of the two distributions of bad borrowers and the bimodal intensity.

\subsubsection{Normal distributions of good and bad borrowers}

The parameters used in the different score simulations and the values obtained for each validation technique used are presented in Table 3.

For the portfolios with normal distributions of good and bad borrowers, it is expected that the indicators would exhibit a decreased discriminative ability when the means of the distributions approach one another or as the distribution deviations increase. From an analysis of Table 3, it is possible to observe that all of the indicators support this claim, that is, as the distribution means get closer or as the distribution deviation increases, a decrease in all indicators can be observed. Therefore, the results suggest that all the metrics present a loss of performance due to the approximation of the probability distributions of good and bad borrowers.

\subsubsection{Normal distribution of good borrowers and bimodal distribution of bad borrowers}

As described earlier, for the simulations with normal distributions of good borrowers and bimodal distributions for bad borrowers, the deviations of all distributions ( Good, $D M_{1}$ and $D M_{2}$ ) were set to 1.0, and the distribution mean of good borrowers was set to 5.0. The parameters that were varied among different distributions were the distribution means of bad borrowers and the ratio of bad borrowers in each of them. Although the mean of the bad borrower distributions was varied, they were equidistant to the distribution mean of good borrowers, as shown in Table 4.

When the performance indicators were analyzed assuming a fixed ratio of bad borrowers for $M_{1}$ and $M_{2}$, that is, by varying only the bad borrower distribution means, we observed a decrease in performance for all indicators because the distributions of bad borrowers were closer to the distribution of good borrowers. This occurrence was expected because the approximation between the means of the distribution will result in a less discriminative ability for the model. However, when ob-

Table 13

CI obtained from simulated portfolios with a correlation of 0.5 between $X_{1}$ and $X_{2}$.

\begin{tabular}{|c|c|c|c|c|c|c|c|c|c|}
\hline Initial sample & KS & AUROC & AR & Pietra Index & Brier & CIER & $\mathrm{KL}$ & IV & M \\
\hline Mean & 0.3023 & 0.6934 & 0.3968 & 0.1069 & 0.0854 & 0.0693 & 0.0224 & 0.5429 & 0.8782 \\
\hline Deviation & 0.0143 & 0.0075 & 0.0153 & 0.0051 & 0.0021 & 0.0055 & 0.0019 & 0.0499 & 0.0387 \\
\hline Lower limit (95\% CI) & 0.1714 & 0.6824 & 0.3648 & 0.0963 & 0.0811 & 0.0577 & 0.0185 & 0.4384 & 0.7972 \\
\hline Upper limit (95\% CI) & 0.3323 & 0.7144 & 0.4288 & 0.1175 & 0.0897 & 0.0808 & 0.0262 & 0.6474 & 0.9592 \\
\hline
\end{tabular}

Table 14

Values obtained by changing the correlation between $X_{1}$ and $X_{2}$.

\begin{tabular}{|c|c|c|c|c|c|c|c|c|c|}
\hline Correlation & KS & AUROC & AR & Pietra Index & Brier & CIER & $\mathrm{KL}$ & IV & M \\
\hline 0.1 & 0.354 & 0.724 & 0.448 & 0.125 & 0.084 & 0.089 & 0.029 & 0.669 & 0.861 \\
\hline 0.2 & 0.364 & 0.736 & 0.473 & 0.129 & 0.087 & 0.1 & 0.033 & 0.79 & 0.82 \\
\hline 0.3 & $0.349^{*}$ & $0.721^{*}$ & $0.442^{*}$ & $0.123^{*}$ & 0.087 & $0.088^{*}$ & 0.029 & $0.654^{*}$ & $0.877^{*}$ \\
\hline 0.4 & $0.33^{*}$ & $0.713^{*}$ & $0.425^{*}$ & $0.117^{*}$ & 0.083 & $0.078^{*}$ & $0.025^{*}$ & $0.591^{*}$ & 0.843 \\
\hline 0.55 & $0.299^{*}$ & 0.699* & 0.399* & $0.106^{*}$ & 0.087 & $0.073^{*}$ & $0.023^{*}$ & $0.592^{*}$ & 0.776 \\
\hline 0.6 & $0.3^{*}$ & $0.692^{*}$ & $0.385^{*}$ & $0.106^{*}$ & 0.086 & $0.064^{*}$ & $0.02^{*}$ & $0.477^{*}$ & 0.76 \\
\hline 0.7 & $0.264^{*}$ & $0.668^{*}$ & $0.336^{*}$ & $0.093^{*}$ & $0.094^{*}$ & $0.05^{*}$ & $0.017^{*}$ & $0.374^{*}$ & $0.663^{*}$ \\
\hline 0.8 & $0.263^{*}$ & $0.677^{*}$ & $0.355^{*}$ & $0.093^{*}$ & $0.09^{*}$ & $0.054^{*}$ & $0.017^{*}$ & $0.411^{*}$ & $0.696^{*}$ \\
\hline 0.9 & $0.244^{*}$ & $0.66^{*}$ & $0.321^{*}$ & $0.086^{*}$ & $0.09^{*}$ & $0.043^{*}$ & $0.014^{*}$ & $0.339 *$ & $0.655^{*}$ \\
\hline
\end{tabular}


Table 15

Estimated/observed values in ratings for different correlations.

\begin{tabular}{|c|c|c|c|c|c|c|c|c|c|c|c|c|c|c|c|c|}
\hline \multirow[t]{2}{*}{ Rating } & \multicolumn{4}{|c|}{ Original (Correl $=0.0$ ) } & \multicolumn{4}{|c|}{ Correl $=0.1$} & \multicolumn{4}{|c|}{ Correl $=0.55$} & \multicolumn{4}{|c|}{ Correl $=0.90$} \\
\hline & $E_{s t_{M}}$ & $E t_{B}$ & $O b s_{M}$ & $\mathrm{Obs}_{B}$ & $E s t_{M}$ & $\mathrm{Est}_{B}$ & $O b s_{M}$ & $\mathrm{Obs}_{B}$ & $E s t_{M}$ & $E s t_{B}$ & $O b s_{M}$ & $\mathrm{Obs}_{B}$ & Est $_{M}$ & Est $_{B}$ & $O b s_{M}$ & $O b s_{B}$ \\
\hline 1 & 276 & 724 & 260 & 740 & 286 & 714 & 263 & 737 & 341 & 659 & 230 & 770 & 400 & 600 & 178 & 822 \\
\hline 2 & 165 & 835 & 191 & 809 & 167 & 833 & 191 & 809 & 190 & 810 & 168 & 832 & 216 & 784 & 145 & 855 \\
\hline 3 & 124 & 876 & 130 & 870 & 124 & 876 & 150 & 850 & 136 & 864 & 136 & 864 & 151 & 849 & 140 & 860 \\
\hline 4 & 98 & 902 & 120 & 880 & 97 & 903 & 91 & 909 & 103 & 897 & 115 & 885 & 112 & 888 & 119 & 881 \\
\hline 5 & 80 & 920 & 68 & 932 & 78 & 922 & 73 & 927 & 79 & 921 & 77 & 923 & 83 & 917 & 98 & 902 \\
\hline 6 & 65 & 935 & 62 & 938 & 62 & 938 & 65 & 935 & 61 & 939 & 100 & 900 & 62 & 938 & 82 & 918 \\
\hline 7 & 52 & 948 & 38 & 962 & 50 & 950 & 51 & 949 & 47 & 953 & 58 & 942 & 46 & 954 & 64 & 936 \\
\hline 8 & 41 & 959 & 39 & 961 & 39 & 961 & 52 & 948 & 34 & 966 & 52 & 948 & 32 & 968 & 57 & 943 \\
\hline 9 & 30 & 970 & 22 & 978 & 28 & 972 & 27 & 973 & 23 & 977 & 25 & 975 & 21 & 979 & 37 & 963 \\
\hline 10 & 17 & 983 & 17 & 983 & 15 & 985 & 23 & 977 & 11 & 989 & 15 & 985 & 10 & 990 & 28 & 972 \\
\hline
\end{tabular}

Table 16

Values obtained by changing the correlation (for the model with a correlation of 0.5 ).

\begin{tabular}{|c|c|c|c|c|c|c|c|c|c|}
\hline Correlation & KS & AUROC & $\mathrm{AR}$ & Pietra Index & Brier & CIER & $\mathrm{KL}$ & IV & M \\
\hline 0.10 & $0.343^{*}$ & $0.722^{*}$ & $0.445^{*}$ & $0.121^{*}$ & 0.084 & $0.088^{*}$ & $0.023^{*}$ & $0.656^{*}$ & $0.762 *$ \\
\hline 0.20 & $0.386^{*}$ & $0.737^{*}$ & $0.473^{*}$ & $0.13^{*}$ & 0.087 & $0.102^{*}$ & $0.034^{*}$ & $0.303^{*}$ & $0.733^{*}$ \\
\hline 0.30 & $0.348^{*}$ & $0.721^{*}$ & $0.441^{*}$ & $0.123^{*}$ & 0.086 & $0.086^{*}$ & $0.023^{*}$ & 0.646 & 0.798 \\
\hline 0.40 & 0.332 & 0.713 & 0.426 & 0.117 & 0.082 & 0.079 & 0.025 & 0.595 & 0.874 \\
\hline 0.55 & 0.300 & 0.699 & 0.398 & 0.106 & 0.085 & 0.072 & 0.023 & 0.58 & 0.869 \\
\hline 0.60 & 0.298 & 0.693 & 0.387 & 0.106 & 0.084 & 0.064 & 0.02 & 0.479 & 0.9 \\
\hline 0.70 & $0.269^{*}$ & $0.668^{*}$ & $0.336^{*}$ & $0.095^{*}$ & $0.091^{*}$ & $0.049^{*}$ & $0.016^{*}$ & $0.371^{*}$ & 0.83 \\
\hline 0.30 & $0.264^{*}$ & $0.677^{*}$ & $0.353^{*}$ & $0.093^{*}$ & 0.086 & $0.054^{*}$ & $0.017^{*}$ & $0.413^{*}$ & 0.874 \\
\hline 0.90 & $0.247^{*}$ & $0.66^{*}$ & $0.321^{*}$ & $0.087^{*}$ & 0.085 & $0.044^{*}$ & $0.014^{*}$ & $0.345^{*}$ & 0.832 \\
\hline
\end{tabular}

serving the effect caused by the increased bimodal characteristic (ratio equalization between $M_{1}$ and $M_{2}$ ), indicators such as $A U R O C, A R, K S$, and Pietra exhibited a significant drop in model performance, which was unexpected because the distributions $M_{1}$ and $M_{2}$ had the same variance and means that were equidistant from the mean value of the good borrower distribution. This decrease in performance can also be observed for the CIER, KL and IV indicators, although the decreases were much less significant for these indicators. By analyzing the results for simulations with mean 7.5 and ratios $90 \%$ and $50 \%$ for $D M_{1}$, it is possible to observe that the KS, AUROC, AR, and Pietra metrics classify the model with a ratio of $90 \%$ as having good performance and the model with a ratio of $50 \%$ as having bad performance; however, CIER, KL and IV classify both as good models. The results show that due to the bimodal feature of bad borrowers, some metrics can better convey information about the quality of the scores. Traditional metrics fail to assess changes in the performance of the discrimination model whereas metrics based on entropy are more sensitive. ${ }^{9}$

\subsection{Portfolios with independent explanatory variables}

For the portfolios with independent explanatory variables, 20 portfolios were simulated and the validation techniques were applied. Thus, it was possible to define the confidence intervals for each technique. The mean value of the metrics and the confidence interval defined are presented in Table 5.

It is important to stress that the model chosen among the 20 models used for the construction of the confidence interval, which was used in other simulations with variation of the mean and variance parameters, was the regression model developed from sample Indep $_{16}(K S=$

\footnotetext{
9 These results demonstrate the importance of validation of DP metrics, as a technique that is unable to properly discriminate different borrowers can produce Type I and Type II errors in terms of good/bad credit. Sobehart et al. (2000a) and Sobehart, Keenan, and Stein (2000b) present an analysis where Type I and Type II errors are discussed: Type I error is associated with a high-credit rating for a bad borrower, which can result in an excess in the number of defaults. Type II error means that a low-credit rating is assigned to a good borrower which is traduced in a lower return given that less number of credits to good borrowers will be assigned as a result of a better bidding rate by the competition. The AUROC measure resulted the best measure in terms of lower Type I and II errors.
}

0.3823, AUROC $=0.7495, A R=0.4989$, Pietra $_{\text {Index }}=0.1352$, Brier $=$ $0.0812, C I E R=0.1141, K L=0.0361, I V=0.9392, M=0.8355)$.

\subsection{Impact caused by variation of the $X_{1}$ mean}

Simulations in which the mean of the independent variable $X_{1}$ was varied were performed, and the chosen model was applied. The results of the metrics obtained using the validation techniques can be found in Table 6.

It is possible to observe from the results presented in Table 6 that with the exception of metric $M$, none of the other metrics exhibited a drop in model performance with displacement of the mean of variable $X_{1}$ to lower or higher values. Metric $M$ exhibited a drop in model performance in both cases, that is, the value of $M$ decreased as the $X_{1}$ mean was increased or decreased. Table 7 presents the estimated and observed values for each rating.

where:

Est $_{M} \quad$ is the number of bad borrowers estimated by the model; Est $_{B} \quad$ is the number of good borrowers estimated by the model; $\mathrm{Obs}_{M}$ is the number of bad borrowers observed in the rating, and. $\mathrm{Obs}_{B} \quad$ is the number of good borrowers observed in the rating.

According to Table 7, there was considerable variation in the value of bad borrowers estimated for each rating. This variation was caused by the change in the mean $X_{1}$ value, i.e., the accuracy of the model was greatly affected by the change in the mean of the independent variables, and this aspect can only be observed in the values of measure $M$. The entropy measures did not undergo considerable changes because the default rate observed for each rating did not vary greatly. The more traditional metrics, such as KS, AUROC, Pietra, and $A R$, remained stable, possibly because the rankings of good and bad borrowers did not undergo major changes. The loss of performance of measure $M$ in Table 6 is a consequence of the lower accuracy of the model. Table 7 corroborates this argument, since the differences between the estimated and the observed values increase with the shift of the mean of the explanatory variable. 
Table 17

Estimated/observed values in ratings (for the model with a correlation of 0.5 ).

\begin{tabular}{|c|c|c|c|c|c|c|c|c|c|c|c|c|c|c|c|c|}
\hline \multirow[t]{2}{*}{ Rating } & \multicolumn{4}{|c|}{ Original (Correl $=0.5$ ) } & \multicolumn{4}{|c|}{ Correl $=0.1$} & \multicolumn{4}{|c|}{ Correl $=0.55$} & \multicolumn{4}{|c|}{ Correl $=0.90$} \\
\hline & $E s t_{M}$ & $E t_{B}$ & $O b s_{M}$ & $\mathrm{Obs}_{B}$ & $E s t_{M}$ & $E s t_{B}$ & $O b s_{M}$ & $O b s_{B}$ & $E s t_{M}$ & $E t_{B}$ & $O b s_{M}$ & $\mathrm{Obs}_{B}$ & $E s t_{M}$ & Est $_{\mathrm{B}}$ & $O b s_{M}$ & $O b s_{B}$ \\
\hline 1 & 250 & 750 & 232 & 768 & 213 & 787 & 263 & 737 & 245 & 755 & 226 & 774 & 279 & 721 & 179 & 821 \\
\hline 2 & 161 & 839 & 169 & 831 & 145 & 855 & 195 & 805 & 159 & 841 & 171 & 829 & 174 & 826 & 146 & 854 \\
\hline 3 & 129 & 871 & 141 & 859 & 119 & 881 & 136 & 864 & 126 & 874 & 138 & 862 & 136 & 864 & 135 & 865 \\
\hline 4 & 107 & 893 & 113 & 887 & 101 & 899 & 95 & 905 & 105 & 895 & 119 & 881 & 111 & 889 & 129 & 871 \\
\hline 5 & 90 & 910 & 109 & 891 & 87 & 913 & 82 & 918 & 88 & 912 & 77 & 923 & 91 & 909 & 94 & 906 \\
\hline 6 & 76 & 924 & 78 & 922 & 75 & 925 & 66 & 934 & 74 & 926 & 93 & 907 & 75 & 925 & 81 & 919 \\
\hline 7 & 64 & 936 & 58 & 942 & 65 & 935 & 50 & 950 & 62 & 938 & 59 & 941 & 62 & 938 & 65 & 935 \\
\hline 8 & 53 & 947 & 41 & 959 & 55 & 945 & 46 & 954 & 51 & 949 & 50 & 950 & 49 & 951 & 53 & 947 \\
\hline 9 & 41 & 959 & 35 & 965 & 44 & 956 & 29 & 971 & 39 & 961 & 28 & 972 & 37 & 963 & 37 & 963 \\
\hline 10 & 25 & 975 & 21 & 979 & 30 & 970 & 24 & 976 & 24 & 976 & 15 & 985 & 22 & 978 & 29 & 971 \\
\hline
\end{tabular}

\subsubsection{Impact caused by changes in the variance of $\mathrm{X}_{2}$}

From an application of the chosen model to samples with variation of the $X_{2}$ variance (originally, the variance had the value 4.0), the results obtained for the validation metrics are presented in Table 8 .

It is possible to observe from the results presented in Table 8 that the measures are, in general, not very sensitive to changes in the variance of the independent variable, and only a major change in the variance (deviation equal to 10.00) caused the indicators to be outside the estimated confidence interval. Regarding the correctness of the default values of the ratings, it is possible to observe a result consistent with other metrics, i.e., a lower correctness for the deviation of 10.00 , as indicated by Table 9 .

\subsubsection{Impact caused by changes to the bi-stochastic matrices}

The matrices initially used to associate variables $X_{1}$ and $X_{2}$ to variable $Y$ were the matrices $M_{1}$ and $M_{1}$. These matrices had high values in their diagonals (the main diagonal in case of $X_{1}$ and the secondary diagonal in case of $X_{2}$ ) that cause a stronger dependency relationship between independent variables and the dependent variable. Table 10 presents the results of the validation techniques obtained using the chosen model for portfolios simulated using the other bi-stochastic matrices.

Based on the results presented in Table 10, it is possible to observe that all metrics except the Brier score exhibited decreased model performance as the dependency between the variables was changed. Measure
$M$ was relatively insensitive to the change in dependency because its value changed less sensitively than those of other measures, such as the entropy measures (CIER, IV, and KL), KS, AUROC, Pietra, and AR. In practical terms, the metrics indicate that if the model was developed using a variable that has a strong relationship with the default event and this relationship is weakened over time, the discriminative ability of the model will decrease. Table 11 presents the estimated and observed values for each model rating.

It is possible to observe from the information contained in Table 11 that there was no considerable change in model correctness between the original portfolio and the portfolio simulated from $M_{3}$ and $M_{3}$. There was a more significant change in model correctness for the portfolio simulated from the matrices $M_{5}$ and $M_{5}$. Another aspect that can be observed is that in the original model, the heterogeneity of the rate of defaults observed among the ratings is greater than in the portfolio that used the matrices $M_{5}$ and $M_{5}$. This greater homogeneity in default rates among ratings can be observed from the sharp drop in the value of the entropy measures among portfolios.

\subsection{Portfolios with dependent variables simulated using gaussian copulas}

Similar to the tests conducted with independent variables, 20 portfolios were generated to determine a confidence interval for the validation techniques. However, in this case, 20 portfolios with zero correlation between $X_{1}$ and $X_{2}$ and 20 portfolios with a correlation of

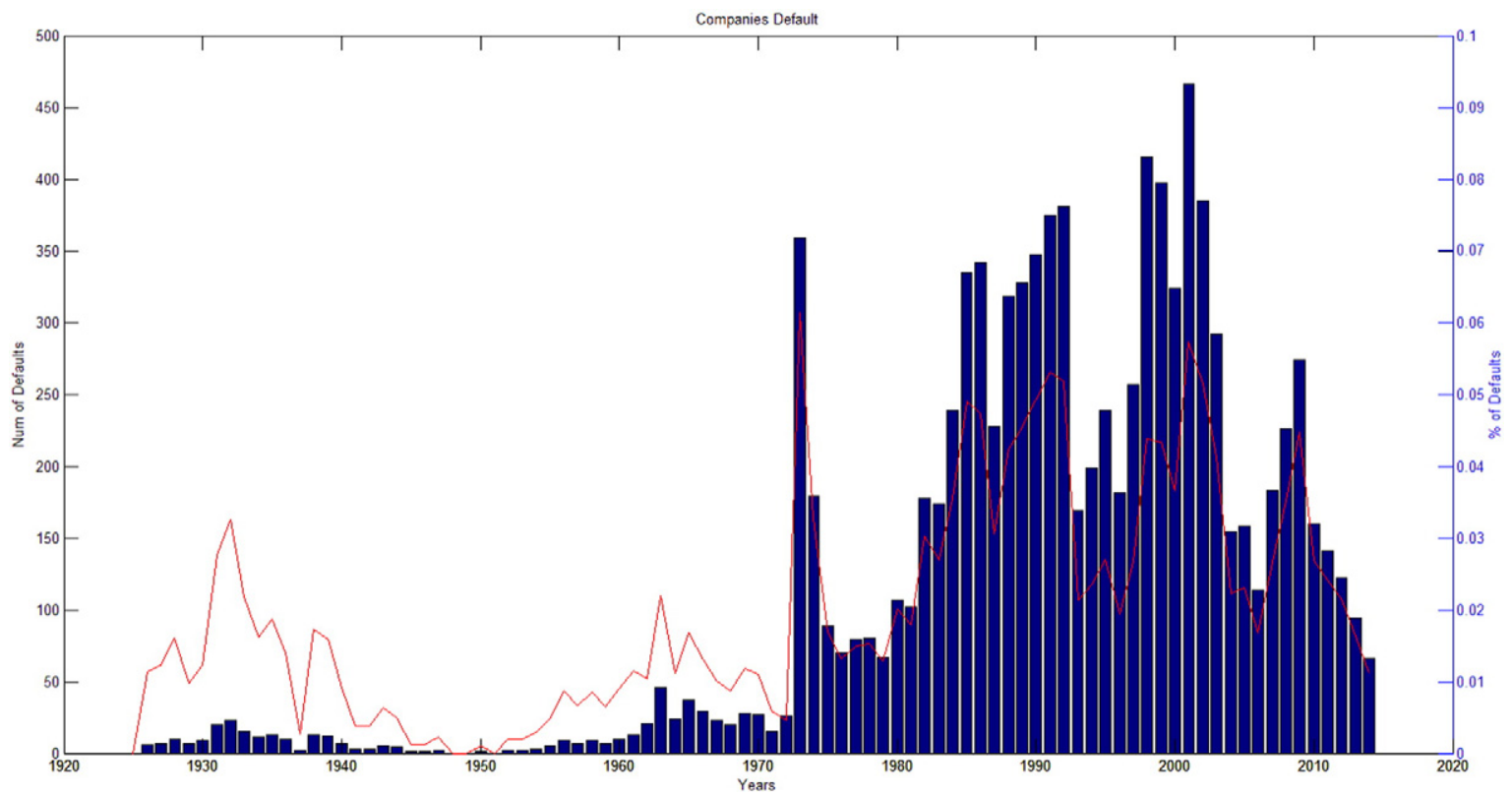

Fig. 2. Number (blue bars) and proportion (red line) of public companies default from 1950 to 2014. Source: CRSP database. 
Table 18

Effects of change in the mean of $X_{1}$ for validation techniques applied to a calculated empirical distribution of defaulting companies 1950-2014.

\begin{tabular}{|c|c|c|c|c|c|c|c|c|c|}
\hline Firm sector & $X_{1}$ mean adjustment & KS & AUROC & AR & Pietra & CIER & $\mathrm{KL}$ & IV & M \\
\hline \multirow[t]{3}{*}{ Public firms (manufacturing) } & Population mean & 0.169 & 0.292 & -0.139 & 0.0598 & 0.188 & 0.13 & 1.28 & 0.589 \\
\hline & Increased & $0.238^{*}$ & $0.4^{*}$ & $-0.2^{*}$ & $0.0842^{*}$ & $0.275^{*}$ & $0.19^{*}$ & $1.94^{*}$ & $0.914^{*}$ \\
\hline & Decreased & $0.241^{*}$ & $0.396^{*}$ & $-0.207^{*}$ & $0.0853^{*}$ & $0.252^{*}$ & $0.174^{*}$ & $1.68^{*}$ & $0.744^{*}$ \\
\hline \multirow[t]{3}{*}{ Public firms without equity market value (manufacturing) } & Population mean & 0.107 & 0.325 & -0.0722 & 0.0377 & 0.16 & 0.105 & 1.13 & 0.416 \\
\hline & Increased & 0.0946 & $0.477^{*}$ & $-0.0468^{*}$ & 0.0335 & $0.268^{*}$ & $0.186^{*}$ & $1.8^{*}$ & $0.741^{*}$ \\
\hline & Decreased & $0.171^{*}$ & $0.453^{*}$ & -0.0948 & $0.0604^{*}$ & 0.179 & 0.111 & 1.28 & $0.538^{*}$ \\
\hline \multirow[t]{3}{*}{ Non-manufacturing } & Population mean & 0.0718 & 0.341 & -0.0399 & 0.0254 & 0.126 & 0.0757 & 0.837 & 0.409 \\
\hline & Increased & $0.102^{*}$ & $0.479^{*}$ & -0.0423 & $0.0361^{*}$ & $0.217^{*}$ & $0.146^{*}$ & $1.41^{*}$ & $0.658^{*}$ \\
\hline & Decreased & 0.093* & $0.469^{*}$ & $-0.0614^{*}$ & $0.0329^{*}$ & $0.14^{*}$ & 0.072 & $0.936^{*}$ & $0.528^{*}$ \\
\hline
\end{tabular}

0.5 between $X_{1}$ and $X_{2}$ were generated. Tables 12 and 13 present the mean values for each technique and the confidence intervals for portfolios with correlations of zero and 0.5 .

\subsubsection{Impact caused by correlation emergence}

Among the 20 models with zero correlation that were used to determine the confidence interval, model $D e p G_{\text {Zero03 }}$, i.e., $(K S=0.3808$, AUROC $=0.7410, A R=0.4820$, Pietra $_{\text {Index }}=0.1346$, Brier $=0.0805$, CIER $=0.1021, K L=0.0320, I V=0.7957, M=0.8610$ ) was chosen to be applied to the samples with correlation variation. The results obtained for the validation techniques for the samples with correlation are presented in Table 14.

All techniques, with the exception of the Brier score, exhibited a decrease in model performance with increased correlation, which was expected because the model was developed using a portfolio for which the correlation between the variables $X_{1}$ and $X_{2}$ was zero. The observed and estimated values for each of the ratings of some models are presented in Table 15.

Based on the results presented in Table 15, it is possible to observe a significant drop in model correctness with increased correlation between variables $X_{1}$ and $X_{2}$. The heterogeneity of the default rates among the ratings also decreased, which made the entropy measures sensitive. In general, the measures indicate that a model developed with non-correlated variables that explain the default event with good performance can have its performance compromised if a correlation between variables comes into existence. The increase in the correlation generated a relevant impact on model correctness. For instance, taking into consideration rating 6 , the observed default rate overcame the expected default rate in $>60 \%$ of cases when the correlation was 0.55 .

\subsubsection{Impact caused by a change in the correlation}

Among the 20 models with a correlation of 0.50 that were used to determine the confidence interval, model $D e p G_{0.5 ; 13}$, i.e., $(K S=$ $0.3005, A U R O C=0.6998, A R=0.3996$, Pietra $a_{\text {Index }}=0.1062$, Brier $=$ $0.0858, C I E R=0.0691, K L=0.0224, I V=0.5365, M=0.8897$ ) was chosen to be applied to the samples with distinct correlations. The results obtained for the validation techniques for these samples are presented in Table 16.

Except for the Brier Score, all measures were sensitive to variations in the correlation. However, because the model was developed with a correlation of 0.5 , a decrease in performance was expected if the correlation values increased or decreased. All sensitive metrics, with the exception of measure $M$, exhibited an increase in model performance as the strength of the correlation was decreased. Measure $M$ exhibited lower model performance for both increased and decreased correlation, although the values were outside the confidence interval only for the decreased correlation. Consequently, Table 17 presents the estimated and observed values of default within the ratings for the models.

According to Table 17, the model correctness decreased as the correlation was increased or decreased, which explains the values of measure $M$ for these cases. The default rates remained heterogenous along the ratings as the correlations were increased or decreased, which explains the small fluctuation in the entropy measures. The KS, AR, AUROC, and Pietra measures were more sensitive to the order of the subjects. Therefore, it can be concluded that although the correlation change decalibrated the probability values that the model calculates, the order of the good and bad subjects was not significantly changed.

\section{Empirical sub-samples test}

In this section, we developed a methodology for testing the adequacy of techniques for the validation of $D P$ with empirical data. The methodology presented in Section 3, where a numerical simulation with control over the variables (factor variables $X_{1}, X_{2}$ and the response variable of default $Y$ ) is used to analyze the effects of the techniques for the validation of $D P$, is useful when we try to understand the effectiveness in ideal situations. In particular, we can study the behavior of metrics due to changes in the distribution, such as when the mean, the variance, and the correlation are changed. However, the values of the input

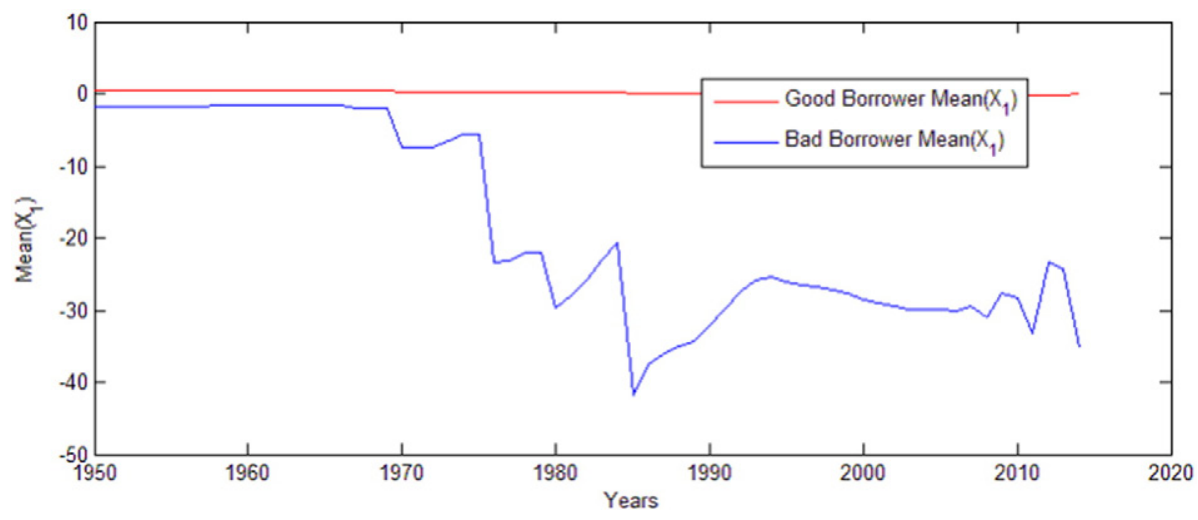

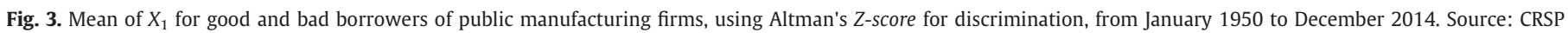
and COMPUSTAT. 
Table 19

Effects of change in the volatility of $X_{1}$ for validation techniques applied to a calculated empirical distribution of defaulting companies $1950-2014$.

\begin{tabular}{|c|c|c|c|c|c|c|c|c|c|}
\hline Firm sector & $X_{1}$ volatility adjustment & KS & AUROC & AR & Pietra & CIER & $\mathrm{KL}$ & IV & M \\
\hline \multirow[t]{3}{*}{ Public firms (manufacturing) } & Population mean & 0.169 & 0.292 & -0.139 & 0.0598 & 0.188 & 0.13 & 1.28 & 0.589 \\
\hline & Increased & $0.268^{*}$ & $0.388^{*}$ & $-0.224^{*}$ & $0.0947^{*}$ & $0.25^{*}$ & $0.172^{*}$ & $1.65^{*}$ & $0.743^{*}$ \\
\hline & Decreased & $0.119^{*}$ & $0.475^{*}$ & $-0.0498^{*}$ & $0.042^{*}$ & $0.27^{*}$ & $0.186^{*}$ & $1.85^{*}$ & $0.824^{*}$ \\
\hline \multirow[t]{3}{*}{ Public firms without equity market value } & Population mean & 0.107 & 0.325 & -0.0722 & 0.0377 & 0.16 & 0.105 & 1.13 & 0.416 \\
\hline & Increased & $0.2078^{*}$ & $0.416^{*}$ & $-0.169^{*}$ & $0.0732^{*}$ & $0.226^{*}$ & 0.134 & $1.8^{*}$ & $0.264^{*}$ \\
\hline & Decreased & 0.147 & $0.421^{*}$ & $-0.159^{*}$ & 0.052 & $0.224^{*}$ & $0.15^{*}$ & $1.51^{*}$ & $0.568^{*}$ \\
\hline \multirow[t]{3}{*}{ Non-manufacturing } & Population mean & 0.0718 & 0.341 & -0.0399 & 0.0254 & 0.126 & 0.0757 & 0.837 & 0.409 \\
\hline & Increased & $0.127^{*}$ & $0.453^{*}$ & $-0.0936^{*}$ & $0.0448^{*}$ & $0.148^{*}$ & $0.0696^{*}$ & $1.03^{*}$ & 0.423 \\
\hline & Decreased & 0.0761 & $0.486^{*}$ & -0.0283 & 0.0269 & $0.188^{*}$ & $0.12^{*}$ & $1.22^{*}$ & $0.571^{*}$ \\
\hline
\end{tabular}

parameters used for the numerical simulation results in Section 4 were not calibrated with market data; they were simply used as a numerical application of the methodology.

The purpose of this section is to provide a real-life application analysing the effects of techniques of validation for different market conditions. Comparing our analysis with that of Sobehart et al. (2000a), the contribution of this section is that we use a larger dataset and our results are provided when controlling factor variables $X_{1}, X_{2}$ by market situations, giving the risk manager an idea of the capacity of the techniques of validation of $D P$ for historical market situations.

\subsection{Methodology}

The first part of the methodology involves defining what is a good borrower and a bad borrower. We also define the variables $X_{1}, X_{2}$, and $Y$. We adopted Altman1968's Altman1968 Z-score to determine which companies are in distress. The Z-score, which was initially developed for public manufacturing firms (Altman, 1968), was extended for private firms and non-manufacturing firms (Altman, 2000). In these three models financial ratios with fundamental balance sheet and income statement data are aggregated in a discriminant analysis study to determine companies in financial distress. There are five ratios in the original study of Altman (1968):

$T_{1} \quad$ Working Capital/Assets;

$\mathrm{T}_{2} \quad$ Retained Earnings/Assets;

$T_{3} \quad$ EBIT/Assets;

$T_{4} \quad$ Market Value of Equity/Liabilities; and,

$T_{5} \quad$ Sales/Assets.

where Assets and Liabilities are totalled and the regression is given by (17), as in Altman (1968):

$Z=1.2 T_{1}+1.4 T_{2}+3.3 T_{3}+0.6 T_{4}+0.99 T_{5}$,
This equation is used only for public manufacturing companies. The modified equation for private manufacturing companies is given by (18) as in Altman (2000):

$Z=1.7 T_{1}^{\prime}+1.8 T_{2}+3.1 T_{3}+0.4 T_{4}^{\prime}+0.99 T_{5}$, where:

$T_{1} \quad$ (Curr Assets - Curr Liabilities) / Assets; and

$T_{4} \quad$ Book Value of Equity / Liabilities.

and for non-manufacturing and emerging market companies it is given by (19) as in Altman (2000):

$Z^{\prime \prime}=6.56 T_{1}^{\prime}+3.26 T_{2}+6.72 T_{3}+1.05 T_{4}^{\prime}$,

Altman (2000) found some critical values $(Z<1.81$ for public manufacturing, $\mathrm{Z}<1.23$ for private manufacturing, and $\mathrm{Z}<1.1$ for non-manufacturing companies). We define a bad borrower to be one with an Altman Z-score that is below the critical level and otherwise it is a good borrower. The factor variables are defined by the ratios, $X_{1}=T_{2}, X_{2}=T_{3}$, and the credit status is $Y=1$ in the event of default and zero otherwise.

Extracting the DP from the market data is a challenging task. As defaults occur during the year, we model the DP using a discrete approach, defining an annualized dynamic model. Then, using an in-sample approach, the distribution of defaults will be such that if the company defaults during a year the values of the ratios $X_{1}$ and $X_{2}$ will be associated with $Y=1$ and with $Y=0$ otherwise.

\subsection{Data}

The data for the defaulting companies were proxied by the information on 30,686 delisted public companies that was extracted from the CRSP database and crossed with the financial fundamentals provided by the COMPUSTAT database from January 1950 to December 2014. Although all the companies were public, some of them do not have

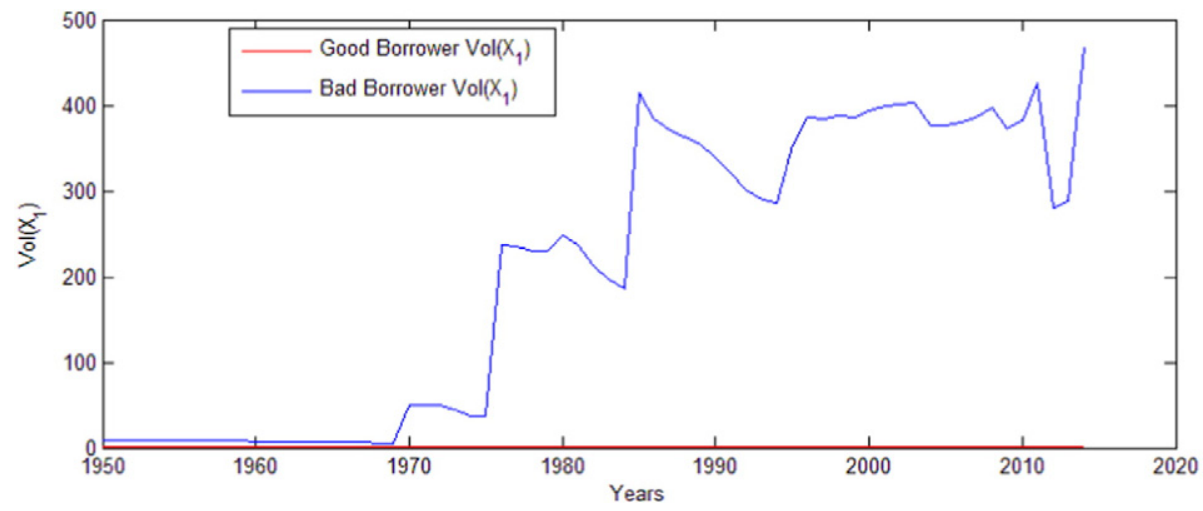

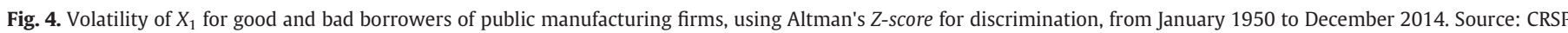
and COMPUSTAT. 


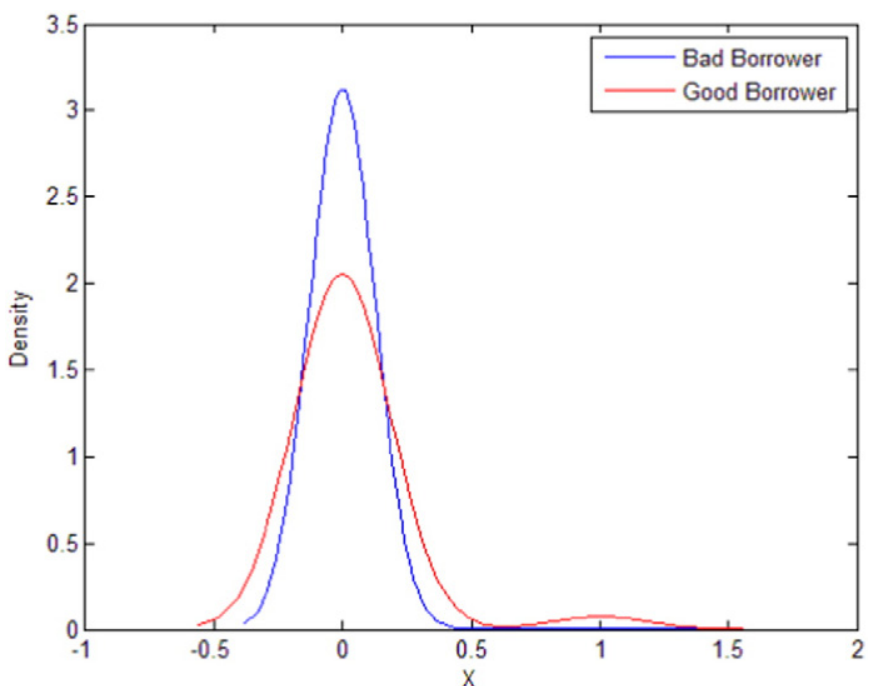

Fig. 5. Empirical distribution of the $X_{1}$ variable for good borrowers (red line) and bad borrowers.

enough liquidity to disclose a market value for their equity; in this case, we used the Altman's Z-score for private firms.

\subsection{Results}

The empirical test for the sensitivity of the validation measures identified $A U R O C$ as the most robust measure to detect changes in the distribution of $D P$. Fig. 2 shows the number of defaults and the proportion of defaults for the 30,686 companies.

\subsubsection{Controlling factor variables}

One of the main contributions of this research is the ability of the methodology to control the factor variables $X_{1}, X_{2}$ when studying the effects of changing conditions over the techniques of the validation of $D P$. We define three axes for the sensitivity analysis of the numerical simulation of Section 4: changes in the mean of $X_{1}$, changes in the volatility of $X_{1}$, and changes in the correlation between $X_{1}$ and $X_{2}$. For a discriminant analysis study we classify the market conditions each year by two groups in high-low $X_{1}$ 50th-percentile mean, high-low $X_{1}$ 50th-percentile volatility and high-low $X_{1}$ vs. $X_{2}$ 50th-percentile pair correlation. We then study the effects of an increase/decrease in the mean of $X_{1}$, controlling for a low percentile volatility and a low percentile correlation.

For example, let each year to be associated with a $Y, X_{1}, X_{2}$ matrix. We split the set of years into two big groups: years with high values (over the 50th-percentile) of the mean of $X_{1}$, and years with low values of the mean of $X_{1}$. We then selected market conditions associated with extreme markets; that is, high volatility and high correlation. The two big groups are reduced by controlling such that the two groups have similar levels of volatility and correlation and differ only in the mean value. We do the same with the volatility and the correlation. With these two smaller groups, we calculate the KS, AROC, AR, Pietra, CIER, KL, and IV measures for each sub-group and then for the whole population. The mean value of $X_{1}$ will then differ between the population, the high mean of the $X_{1}$ group and the low mean of the $X_{1}$ group.

\subsubsection{Impact caused by variation of the $X_{1}$ mean}

Table 18 shows the effects of changing the mean of $X_{1}$ over the techniques for the validation of $D P$. For public firms, an increase and decrease of the mean of $X_{1}$ was recognized by all measures. Fig. 3 shows the mean of $X_{1}$ for good and bad borrowers from 1950 to 2014. It is evident that the variable selected for the variable $X_{1}\left(T_{2}\right)$ discriminates properly in terms of the mean of the two groups. In the case of the public firms without a market value, the years with lower 50th-percentile mean values were not detected for the $A R, C I E R, K L$, and IV measures. For the non-manufacturing firms, $A R$ and $K L$ did not detect the change in the mean. AUROC and measure $M$ proved to be the most sensitive measures in all cases.

\subsubsection{Impact caused by changes in the variance of $X_{1}$}

Table 19 shows the effects of changing volatility. For public firms, all measures captured the changes correctly. Nevertheless, KS, AR, Pietra, and $K L$ show decreases in performance in the case of private manufacturing and non-manufacturing firms. AUROC proved to be the best measure.

Comparing the volatility of $X_{1}$ for good and bad borrowers through the years, Fig. 4 shows a sudden increase during the latter years for the distressed companies. When comparing the empirical distribution of $X_{1}$ for good borrowers and bad borrowers with Fig. 5, we notice that although there is a difference in the mean and the volatility, it is not possible to perform a simple discrimination of samples from both populations from the distribution given that both distributions overlap.

\subsubsection{Impact caused by changes in the pair correlation of $X_{1}$ and $X_{1}$}

As shown in Table 20, identifying changes in the correlation between $X_{1}$ and $X_{1}$ for validation measures was more difficult than for the mean and volatility. Correlation is a more complex characteristic of the distribution, and in Fig. 6 we can see that from the 1990s until the 2010s the difference in correlation of $X_{1}$ and $X_{2}$ between good and bad borrowers has not been a good discriminant. This result has impacts on the techniques of the validation of $D P$. Even in this context, $A U R O C$ and measure $M$ again proved to be the best measures.

\section{Conclusions}

The techniques examined in this study evaluate different aspects of the models. Metrics traditionally used in the market, e.g., the KS statistic and AUROC, allow for the evaluation of the order of good and bad borrowers. However, these more traditional metrics are not adequate for assessing changes in the heterogeneity of default rates over the ratings, which are better evaluated using entropy measures. Metric $M$, unlike all of the others investigated, was quite sensitive to the accuracy of the estimated number of defaults in each rating. The empirical analysis

Table 20

Effects of change in the pair correlation between $X_{1}$ and $X_{2}$ for validation techniques applied to a calculated empirical distribution of defaulting companies 1950-2014.

\begin{tabular}{|c|c|c|c|c|c|c|c|c|c|}
\hline Firm sector & $\boldsymbol{X}_{1}, \boldsymbol{X}_{2}$ correlation adjustment & KS & AUROC & AR & Pietra & CIER & $\mathrm{KL}$ & IV & M \\
\hline \multirow[t]{3}{*}{ Public (manufacturing) } & Population mean & 0.169 & 0.292 & -0.139 & 0.0598 & 0.188 & 0.13 & 1.28 & 0.589 \\
\hline & Increased & $0.214^{*}$ & $0.415^{*}$ & -0.169 & $0.0758^{*}$ & $0.255^{*}$ & $0.175^{*}$ & $1.71^{*}$ & $0.792^{*}$ \\
\hline & Decreased & $0.263^{*}$ & $0.389 *$ & $-0.221^{*}$ & $0.093^{*}$ & $0.264^{*}$ & $0.182^{*}$ & $1.82^{*}$ & $0.851^{*}$ \\
\hline \multirow[t]{3}{*}{ Public firms without equity market value (manufacturing) } & Population mean & 0.107 & 0.325 & -0.0722 & 0.0377 & 0.16 & 0.105 & 1.13 & 0.416 \\
\hline & Increased & $0.166^{*}$ & $0.443^{*}$ & $-0.114^{*}$ & $0.0587^{*}$ & 0.189 & 0.12 & 1.33 & $0.545^{*}$ \\
\hline & Decreased & 0.129 & $0.458^{*}$ & -0.0838 & 0.0455 & $0.255^{*}$ & $0.17^{*}$ & $1.8^{*}$ & $0.597^{*}$ \\
\hline \multirow[t]{3}{*}{ Non-manufacturing } & Population mean & 0.0718 & 0.341 & -0.0399 & 0.0254 & 0.126 & 0.0757 & 0.837 & 0.409 \\
\hline & Increased & $0.0899^{*}$ & $0.472^{*}$ & -0.0563 & $0.0318^{*}$ & $0.147^{*}$ & 0.0791 & $0.98^{*}$ & $0.527^{*}$ \\
\hline & Decreased & $0.107^{*}$ & $0.473^{*}$ & -0.0532 & $0.038^{*}$ & $0.202^{*}$ & $0.13^{*}$ & $1.33^{*}$ & $0.608^{*}$ \\
\hline
\end{tabular}




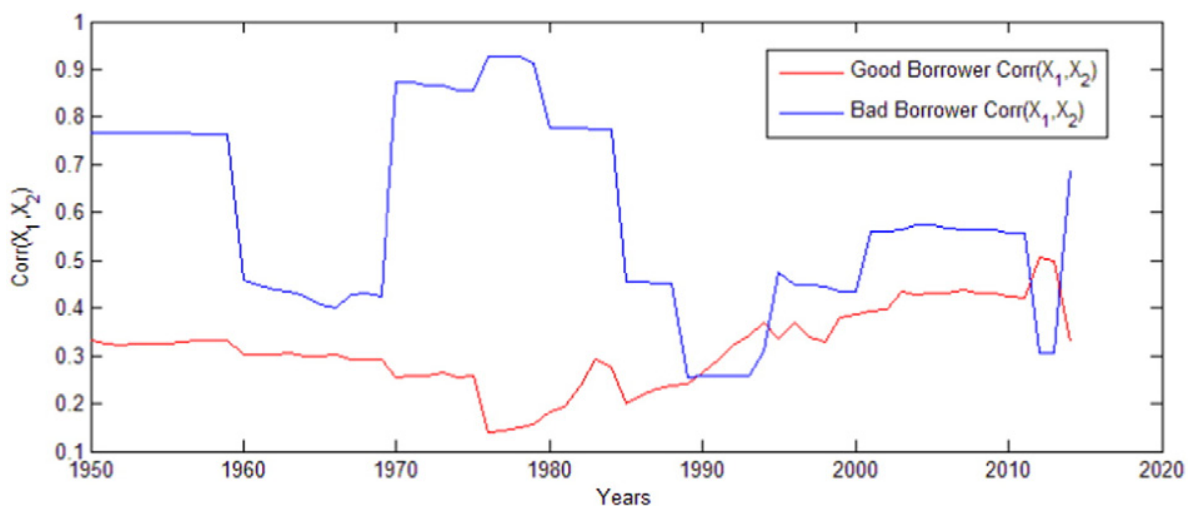

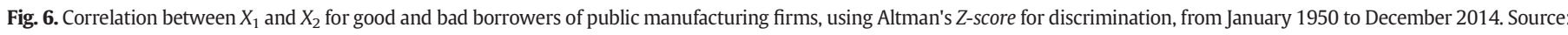
CRSP and COMPUSTAT.

suggests that changes in market conditions are better absorbed by AUROC. However, no validation technique was able to capture all of the impacts generated by changes throughout the tests performed. The techniques are therefore complementary, and none of the techniques can, a priori, be chosen over the others.

The validation techniques presented have been applied in different situations to check the conditions under which a particular technique was more appropriate than the others. Traditional empirical analysis based on a single sample allows one to study a specific market condition, but it is not possible to study the effects that arise from changes in certain parameters. For this purpose, simulated portfolios and controlled empirical samples were used in the stress testing analysis.

First, the distributions of good and bad borrowers along a score scale were simulated. For normal distributions of good and bad borrowers, all the techniques analyzed were effective, i.e., greater similarity of the distributions resulted in decreased performance of all techniques. However, when the simulations were performed using a normal distribution for good borrowers and a bimodal distribution for bad borrowers, it was observed that most traditional techniques were not adequate when the bimodal intensity was high. In these cases, entropy measures were more appropriate. These results are in accordance with those of Engelmann et al. (2003). For portfolios with independent explanatory variables, the decreases in the model accuracy generated by changes in the mean values of the variables were captured only by Measure $M$, and the other measures were not very sensitive. For portfolios with dependent explanatory variables determined using Gaussian copulas, if the model was developed without dependency and dependency came into existence, all metrics except the Brier score exhibited a decrease in model performance.

Second, an empirical analysis with sub-samples of 30,686 delisted US companies was provided, to stress test the validation of $D P$ methodologies. The results were similar to the results found with the numerical simulation, having the AUROC and measure $M$ the most sensitive metrics to changes in the $D P$ distribution main properties (expected return, volatility, and correlation).

Other possibilities for future studies is to investigate other forms of dependency among explanatory variables. Other suggestions for future studies include using other types of explanatory variables (binary, categorical, and beta, for example), changing the distribution used for the definition of the default event, and developing scores from other models, such as decision trees or discriminative analysis, for instance.

\section{Acknowledgments}

This research was supported in part by a grant from the Brazilian National Research Council (CNPq).

\section{Appendix A. Supplementary data}

Supplementary data to this article can be found online at http://dx. doi.org/10.1016/j.irfa.2016.06.007.

\section{References}

Abou-El-Sood, H. (2015 Dec). Are regulatory capital adequacy ratios good indicators of bank failure? Evidence from us banks. International Review of Financial Analysis. http://dx.doi.org/10.1016/j.irfa.2015.11.011.

Agarwal, V., \& Taffler, R. (2008 Aug). Comparing the performance of market-based and accounting-based bankruptcy prediction models. Journal of Banking \& Finance, 32(8), 1541-1551.

Alexander, C., \& Leontsinis, S. (2011). Model Risk in Variance Swap Rates. SSRN Electronic Journal, 1(1), 1-25 ICMA Centre Discussion Papers in Finance DP2011-10.

Alexander, C., \& Sarabia, J. M. (2012 May). Quantile Uncertainty and Value-at-Risk Model Risk. Risk Analysis, 32(8), 1293-1308.

Alexander, C., \& Sheedy, E. (2008 Oct). Developing a stress testing framework based on market risk models. Journal of Banking \& Finance, 32(10), 2220-2236.

Altman, E. I. (1968 Sep). Financial Ratios, Discriminant Analysis and the Prediction of Corporate Bankruptcy. The Journal of Finance, 23(4), 589-609.

Altman, E. I. (2000). Prediciting financial distress of companies: Revisiting the z-score and zeta models. Tech. rep. Working paper. New York University: Department of Finance.

Arnold, B. R., Borio, C., Ellis, L., \& Moshirian, F. (2012 Dec). Systemic risk, Basel III, globa financial stability and regulation. Journal of Banking E' Finance, 36(12), 3123-3124.

Baesens, B., Van Gestel, T., Viaene, S., Stepanova, M., Suykens, J., \& Vanthienen, J. (2003 Jun). Benchmarking state-of-the-art classification algorithms for credit scoring. The Journal of the Operational Research Society, 54(6), 627-635.

BCBS (2005a). Studies on the Validation of Internal Rating Systems. Tech. rep. Basel: Bank for International Settlements, working paper n. 14 of Basel Committee on Banking Supervision.

BCBS (2005b). Studies on the validation of internal rating systems. Tech. rep. Basel: Committee on Banking Supervision - Bank of International Settlements.

BCBS (2006). International convergence of capital measurement and capiatl standards: A revised framework comprehensive version. Tech. rep. Basel Committee on Banking Supervision - Bank of International Settlements.

BCBS (2011). Basel III: A global regulatory framework for more resilient banks and banking systems (revised version). Tech. rep. Basel Committee on Banking Supervision - Bank of International Settlements.

Bensic, M., Sarlija, N., \& Zekic-Susac, M. (2005 Jul). Modelling small-business credit scoring by using logistic regression, neural networks and decision trees. Intelligent Systems in Accounting, Finance and Management, 13(3), 133-150.

Blöchlinger, A. (2012 Jun). Validation of default probabilities. Journal of Financial and Quantitative Analysis, 47(05), 1089-1123.

Boucher, C. M., Daníelsson, J., Kouontchou, P. S., \& Maillet, B. B. (2014 Jul). Risk models-atrisk. Journal of Banking E' Finance, 44(1), 72-92.

Brier, G. W. (1950 January). Verification of forecasts expressed in terms of probability Monthly Weather Review, 78(1), 1-3.

Colletaz, G., Hurlin, C., \& Pérignon, C. (2013 Oct). The Risk Map: A new tool for validating risk models. Journal of Banking \&' Finance, 37(10), 3843-3854.

Davis, J. P., Eisenhardt, K. M., \& Bingham, C. B. (2007 Apr). Developing theory through simulation methods. Academy of Management Review, 32(2), 480-499.

Eliazar, I. I., \& Sokolov, I. M. (2010 Jan). Measuring statistical heterogeneity: The pietra index. Physica A: Statistical Mechanics and its Applications, 389(1), 117-125.

Engelmann, B., Hayden, E., \& Tasche, D. (2003 Jan). Testing rating accuracy. Risk Magazine, $1(1), 1-6$.

Hagedoorn, J. (1996 Jan). Innovation and entrepreneurship: Schumpeter revisited. Industrial and Corporate Change, 5(3), 883-896.

Hakenes, H., \& Schnabel, I. (2011 Jun). Bank size and risk-taking under Basel II. Journal of Banking \& Finance, 35(6), 1436-1449.

Hand, D. J. (2009 Jun). Measuring classifier performance: A coherent alternative to the area under the ROC curve. Machine Learning, 77(1), 103-123. 
Hanley, J. A., \& McNeil, B. J. (1982 Apr). The meaning and use of the area under a receiver operating characteristic (ROC) curve. Radiology, 143(1), 29-36.

Hlawatsch, S., \& Ostrowski, S. (2011). Simulation and estimation of loss given default Journal of Credit Risk, 7(3), 39-73.

Izzi, L., Oricchio, G., \& Vitale, L. (2012). Basel III credit rating systems: An applied guide to quantitative and qualitative models. Palgrave Macmillan.

Jaynes, E. (1957 May). Information Theory and Statistical Mechanics. Physical Review, 106(4), 620-630.

Jobst, N. J., \& Zenios, S. A. (2005 Mar). On the simulation of portfolios of interest rate and credit risk sensitive securities. European Journal of Operational Research, 161(2), 298-324.

Joseph, M. P. (2005 September). A PD Validation Framework for Basel II Internal RatingsBased Systems. Tech. rep. Common Wealth Bank of Australia.

Kalkbrener, M., Lotter, H., \& Overbeck., L. (2004 January). Sensible and efficient allocation for credit portfolios. Risk, 17, S19-S24.

Karakoulas, G. (2004 September). Empirical validation of retail credit-scoring models. The RMA Journal, 1(1), 56-60.

Keenan, S. C., \& Sobehart, J. R. (1999 October). Performance measures for credit risk models Tech. rep. Moody's Risk Management Sevices.

Kerkhof, J., \& Melenberg, B. (2004 Aug). Backtesting for risk-based regulatory capital. Journal of Banking \&' Finance, 28(8), 1845-1865.

Kiefer, N. M. (2009 Jan). Default estimation for low-default portfolios. Journal of Empirical Finance, 16(1), 164-173.

Lilliefors, H. W. (1967 Jun). On the Kolmogorov-Smirnov test for normality with mean and variance unknown. Journal of the American Statistical Association, 62(318), 399-402.

Lopez, J. A., \& Saidenberg, M. R. (2000 Jan). Evaluating credit risk models. Journal of Banking E' Finance, 24(1-2), 151-165.

Marshall, A., Tang, L., \& Milne, A. (2010 Jun). Variable reduction, sample selection bias and bank retail credit scoring. Journal of Empirical Finance, 17(3), 501-512.
Medema, L., Koning, R. H., \& Lensink, R. (2009 Apr). practical approach to validating a PD model. Journal of Banking \& Finance, 33(4), 701-708.

Merton, R. C. (1974 May). On the pricing of corporate debt: The risk structure of interest rates. The Journal of Finance, 29(2), 449-470.

Nelsen, R. B. (1999). An introduction to copulas. New York: Springer.

O'Connell, A A (2006). Logistic Regression Models for Ordinal Response Variables. Vol. 146 of Quantitative Applications in the Social Sciences. SAGE Publications.

Ostrowski, S., \& Reichling, P. (2011 Jun). Measures of predictive success for rating functions. Journal of Risk Model Validation, 5(2), 61-78.

Pietra, G. (1915). Lettere ed arti, tomo lxxiv. Atti del Reale Istituto Veneto di Scienze, 2, 770.

Shumway, T. (2001 January). Forecasting bankruptcy more accurately: A simple hazard model. The Journal of Business, 74(1), 101-124.

Sobehart, J., Keenan, S., \& Stein, R. (2000a). Validation methodologies for default risk models. Credit Magazine, 1(4), 51-56.

Sobehart, J. R. Keenan, S. C., \& Stein, R. M. (2000 marchb). Benchmarking quantitative default risk models: A validation methodology. Tech. rep. Moody's Investors Service.

Steenackers, A., \& Goovaerts, M. (1989 Mar). A credit scoring model for personal loans. Insurance: Mathematics and Economics, 8(1), 31-34.

Stein, R. M. (2007). Benchmarking default prediction models: Pitfalls and remedies in model validation. Journal of Risk Model Validation, 1(1), 77-113.

Tasche, D. (2006). Rating and probability of default validation. Tech. rep. Basel: Bank for International Settlements, working paper n. 14 of Basel Committee on Banking Supervision.

Verbraken, T. Bravo, C. Weber R \& Baesens, B. (2014 Oct). Development and application of consumer credit scoring models using profit-based classification measures. European Journal of Operational Research, 238(2), 505-513.

Zott, C. (2003). Dynamic capabilities and the emergence of intraindustry differential firm performance: Insights from a simulation study. Strategic Management Journal, 24(2), 97-125. 